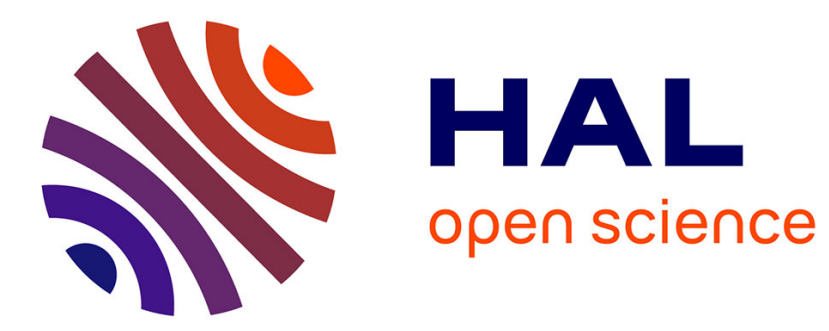

\title{
Multipoint formulas for phase recovering from phaseless scattering data
}

Roman Novikov

\section{To cite this version:}

Roman Novikov. Multipoint formulas for phase recovering from phaseless scattering data. Journal of Geometric Analysis, 2021, 31 (2), pp.1965-1991. 10.1007/s12220-019-00329-6 . hal-02119523

\section{HAL Id: hal-02119523 \\ https://hal.science/hal-02119523}

Submitted on 3 May 2019

HAL is a multi-disciplinary open access archive for the deposit and dissemination of scientific research documents, whether they are published or not. The documents may come from teaching and research institutions in France or abroad, or from public or private research centers.
L'archive ouverte pluridisciplinaire HAL, est destinée au dépôt et à la diffusion de documents scientifiques de niveau recherche, publiés ou non, émanant des établissements d'enseignement et de recherche français ou étrangers, des laboratoires publics ou privés. 


\section{Multipoint formulas for phase recovering from phaseless scattering data}

\section{R.G. Novikov}

CNRS (UMR 7641), Centre de Mathématiques Appliquées, Ecole Polytechnique, Université Paris-Saclay, 91128 Palaiseau, France;

IEPT RAS, 117997 Moscow, Russia

e-mail: novikov@cmap.polytechnique.fr

Abstract. We give formulas for phase recovering from appropriate monochromatic phaseless scattering data at $2 n$ points in dimension $d=3$ and in dimension $d=2$. These formulas are recurrent and explicit and their precision is proportional to $n$. By this result we continue studies of [Novikov, Bull.Sci.Math. 139, 923-936, 2015], where formulas of such a type were given for $n=1, d \geq 2$.

Keywords: Schrödinger equation, Helmholtz equation, Monochromatic scattering data, Phase recovering, Phaseless inverse scattering

AMS subject classification: 35J10, 35P25, 35R30, 81U40

\section{Introduction}

We consider monochromatic wave propagation modelled by the equation:

$$
-\Delta \psi+v(x) \psi=E \psi, \quad x \in \mathbb{R}^{d}, d \geq 1, E>0,
$$

where $\Delta$ is the Laplacian in $x, v$ is a coefficient, e.g., such that

$$
\begin{aligned}
& v \in L^{\infty}\left(\mathbb{R}^{d}\right) \text {, supp } v \subset D, \\
& D \text { is an open bounded domain in } \mathbb{R}^{d} .
\end{aligned}
$$

Equation (1.1) can be considered as the Schrödinger equation at fixed energy $E$ that describes a quantum mechanical particle interacting with a macroscopic object contained in $D$. In this case $v$ is the potential of this interaction.

Equation (1.1) can be also considered as the Helmholtz equation of electrodynamic or acoustic wave propagation at fixed frequency $\omega$. In this case

$$
v(x)=(1-(n(x)))^{2}\left(\frac{\omega}{c_{0}}\right)^{2}, \quad E=\left(\frac{\omega}{c_{0}}\right)^{2},
$$

where $n(x)$ is a scalar index of refraction, $n(x) \equiv 1$ on $\mathbb{R}^{d} \backslash D, c_{0}$ is a reference speed of wave propagation.

For equation (1.1) we consider the classical scattering solutions $\psi^{+}$satisfying the Lippmann-Schwinger integral equation

$$
\begin{aligned}
& \psi^{+}(x, k)=e^{i k x}+\int_{D} G^{+}(x-y, k) v(y) \psi^{+}(y, k) d y, \\
& G^{+}(x, k) \stackrel{\text { def }}{=}-(2 \pi)^{-d} \int_{\mathbb{R}^{d}} \frac{e^{i \xi x} d \xi}{\xi^{2}-k^{2}-i 0}=G_{0}^{+}(|x|,|k|),
\end{aligned}
$$


where $x \in \mathbb{R}^{d}, k \in \mathbb{R}^{d}, k^{2}=E$. Note that

$$
G^{+}(x, k)=-\frac{i}{4} H_{0}^{1}(|x||k|) \text { for } d=2, \quad G^{+}(x, k)=-\frac{e^{i|k||x|}}{4 \pi|x|} \text { for } d=3,
$$

where $H_{0}^{1}$ is the Hankel function of the first type.

Remark 1.1. In the present work, in addition to (1.2), we assume that

equation (1.4) is uniquely solvable for $\psi^{+}(\cdot, k) \in L^{\infty}\left(\mathbb{R}^{d}\right)$ for fixed $E>0$,

where $k \in \mathbb{R}^{d}, k^{2}=E$. If, for example, $v$ satisfies (1.2) and is real-valued, then (1.7) is fulfilled automatically.

In particular, the solutions $\psi^{+}$have the following asymptotics as $|x| \rightarrow \infty$ :

$$
\psi^{+}(x, k)=e^{i k x}+\frac{e^{i|k||x|}}{|x|^{(d-1) / 2}} f_{1}\left(k,|k| \frac{x}{|x|}\right)+O\left(\frac{1}{|x|^{(d+1) / 2}}\right),
$$

where $x \in \mathbb{R}^{d}, k \in \mathbb{R}^{d}, k^{2}=E$. In addition,

$$
\begin{aligned}
& f_{1}(k, l)=c(d,|k|) f(k, l), \\
& c(d,|k|)=-\pi i(-2 \pi i)^{(d-1) / 2}|k|^{(d-3) / 2}, \\
& f(k, l)=(2 \pi)^{-d} \int_{D} e^{-i l y} v(y) \psi^{+}(y, k) d y,
\end{aligned}
$$

where $k \in \mathbb{R}^{d}, l \in \mathbb{R}^{d}, k^{2}=l^{2}=E$.

We recall that $\psi^{+}(x, k)$ at fixed $k$ describes scattering of the incident plane wave described by $e^{i k x}$ on the scatterer described by $v$. In addition, the second term on the right-hand side of (1.8) associates with the leading scattered spherical wave.

The function $f$ arising in (1.8), (1.9) is the classical scattering amplitude for equation (1.1). This function is defined on

$$
\Omega_{E}=\left\{k \in \mathbb{R}^{d}, \quad l \in \mathbb{R}^{d}, \quad k^{2}=l^{2}=E\right\}=\mathbb{S}_{\sqrt{E}}^{d-1} \times \mathbb{S}_{\sqrt{E}}^{d-1},
$$

where

$$
\mathbb{S}_{r}^{d-1}=\left\{m \in \mathbb{R}^{d}: \quad|m|=r\right\} .
$$

We recall that in quantum mechanics, by Born's principle, the values of the functions $\psi^{+}$and $f$ with phase have no direct physical sense, whereas the phaseless values of $\left|\psi^{+}\right|^{2}$ and $|f|^{2}$ have probabilistic interpretations and can be directly obtained in experiments; see $[\mathrm{B}],[\mathrm{FM}]$. On the other hand, in electrodynamic or acoustic experiments $\psi^{+}$and $f$ can be directly measured, at least, in principle. However, in many important cases of monochromatic electro-magnetic wave propagation (e.g., $X$-rays and lasers) the wave frequency is so great that only intensities like $\left|\psi^{+}\right|^{2}$ and $|f|^{2}$ can be measured in practice by modern technical devices; see $[\mathrm{HN}]$ and references therein. 
Multipoint formulas for phase recovering

In the present work we continue studies on the problem of finding $f$ and $\psi^{+}$from $\left|\psi^{+}\right|^{2}$, where $\psi^{+}$and $\left|\psi^{+}\right|^{2}$ are considered outside of $D$.

In particular, in the present work, for fixed $(k, l) \in \Omega_{E}, k \neq l$, for $d=3$ and $d=2$, we give

$$
\begin{aligned}
& \text { formulas for finding } f(k, l) \text { up to } O\left(s^{-n}\right) \text { as } s \rightarrow+\infty \\
& \text { from }\left|\psi^{+}(x, k)\right|^{2} \text { given at } 2 n \text { points } x=x_{1}(s), \ldots, x_{2 n}(s) \text {, }
\end{aligned}
$$

where

$$
\begin{aligned}
& x_{i}(s)=r_{i}(s) \hat{l}, \quad i=1, \ldots, 2 n, \quad \hat{l}=l /|l|, \\
& r_{2 j-1}(s)=\lambda_{j} s, \quad r_{2 j}(s)=\lambda_{j} s+\tau, \quad j=1, \ldots, n, \\
& \lambda_{1}=1, \quad \lambda_{j_{1}}<\lambda_{j_{2}} \text { for } j_{1}<j_{2}, \quad \tau=\tau_{\text {fixed }}>0 .
\end{aligned}
$$

These formulas for $f$ are recurrent and explicit and are presented in detail in Sections 3, $6,7,9,10,11$. The precision of these formulas is $O\left(s^{-n}\right), s \rightarrow+\infty$, and in this sense is proportional to $n$.

In the present work, for fixed $(k, l) \in \Omega_{E}, k \neq l$, for $d=3$ and $d=2$, we also give

$$
\begin{aligned}
& \text { formulas for finding } \psi^{+}(s \hat{l}, k) \text { up to } O\left(s^{-n-(d-1) / 2}\right) \text { as } s \rightarrow+\infty \\
& \text { from }\left|\psi^{+}(x, k)\right|^{2} \text { given at } x=x_{1}(s), \ldots, x_{2 n}(s),
\end{aligned}
$$

where $\hat{l}$ and $x_{i}(s), i=1, \ldots, 2 n$, are defined in (1.15). These formulas for $\psi^{+}$are presented in detail in Sections 3, 12.

Actually, in the present work we continue studies of [N2], [N3], [N4]. In [N2], [N4] formulas of the type (1.14) were given for the 2-point case (i.e., for $n=1$ ) for $d \geq 2$ (but with less precise $O\left(s^{-1 / 2}\right)$ in place of $O\left(s^{-1}\right)$ for $\left.d=2\right)$; see Section 2. In turn, article [N3] gives exact versions (without error terms) of formulas (1.14), (1.16) for the 3-point case for $d=1$; see [N3], [N4] for details.

In addition, the present work continues studies on inverse wave propagation problems without phase information; see Chapter 10 of [ChS], [JL], [IK], [K1], [N2], [N3], [N4], [KR], $[\mathrm{R}],[\mathrm{K} 2],[\mathrm{AHN}],[\mathrm{HN}],[\mathrm{P}]$ and references therein in connection with results given in the literature on the later problem. In particular, formulas (1.14) of the present work and known methods of inverse scattering from $f$ (see [ChS], [N1], [N2] and references therein) yield new results on phaseless inverse scattering from $\left|\psi^{+}\right|^{2}$ measured outside of $D$ for wave propagation modelled by equation (1.1).

Remark 1.2. The approach of the present work as well as of [N2] can be also used for phaseless inverse scattering for obstacles.

2. Two-point formulas for $f$ for $d \geq 2$

We define

$$
a(x, k)=|x|^{(d-1) / 2}\left(\left|\psi^{+}(x, k)\right|^{2}-1\right), \quad x \in \mathbb{R}^{d} \backslash\{0\}, \quad k \in \mathbb{R}^{d} \backslash\{0\},
$$

where $\psi^{+}$is the function of (1.4), (1.8). 
R.G. Novikov

The following formulas of [N2], [N4] hold:

$$
\begin{aligned}
& \left(\begin{array}{l}
\operatorname{Re} f_{1}(k, l) \\
\operatorname{Im} f_{1}(k, l)
\end{array}\right)=M\left(\left(\begin{array}{c}
a\left(x_{1}, k\right) \\
a\left(x_{2}, k\right)
\end{array}\right)-\left(\begin{array}{c}
\delta_{1} a\left(x_{1}, k\right) \\
\delta_{1} a\left(x_{2}, k\right)
\end{array}\right)\right), \\
& M=\frac{1}{2 \sin \left(\varphi_{2}-\varphi_{1}\right)}\left(\begin{array}{cc}
\sin \varphi_{2} & -\sin \varphi_{1} \\
-\cos \varphi_{1} & \cos \varphi_{2}
\end{array}\right), \\
& x_{1}=s \hat{l}, \quad x_{2}=(s+\tau) \hat{l}, \hat{l}=l /|l|, \\
& \varphi_{j}=|k|\left|x_{j}\right|-k x_{j}, \quad j=1,2, \\
& \varphi_{2}-\varphi_{1}=\tau(|k|-k \hat{l}), \\
& \delta_{1} a\left(x_{1}, k\right)=O\left(s^{-\sigma}\right), \quad \delta_{1} a\left(x_{2}, k\right)=O\left(s^{-\sigma}\right) \text { as } s \rightarrow+\infty
\end{aligned}
$$

uniformly in $\hat{k}=k /|k|, \hat{l}=l /|l|$ and $\tau$ at fixed $E>0$,

$$
\sigma=1 / 2 \text { for } d=2, \quad \sigma=1 \text { for } d \geq 3
$$

where

$$
\sin \left(\varphi_{2}-\varphi_{1}\right) \neq 0
$$

$k \in \mathbb{R}^{d}, \quad l \in \mathbb{R}^{d}, \quad|k|=|l|=\sqrt{E}, \quad s>0, \tau>0$.

In view of (1.9), formulas (2.1)-(2.8) are explicit asymptotic formulas for finding phased $f(k, l)$ at fixed $(k, l) \in \Omega_{E}, k \neq l$, from phaseless $\left|\psi^{+}(x, k)\right|^{2}$ at two points $x=x_{1}, x_{2}$ defined in (2.4), where $s \rightarrow+\infty$. For $d \geq 3$ these formulas can be considered as formulas (1.14) for $n=1$. For $d=2$ these formulas can be also considered as formulas of the type (1.14) but with less precise $O\left(s^{-1 / 2}\right)$ in place of $O\left(s^{-1}\right)$.

We recall that (see $[\mathrm{N} 2])$ :

$$
a(x, k)=a_{1}(x, k)+\delta_{1} a(x, k),
$$

where

$$
\begin{aligned}
& a_{1}=2 \operatorname{Re} e^{i \varphi} f_{1} \\
& \delta_{1} a=|x|^{-(d-1) / 2}\left|f_{1}\right|^{2}+2|x|^{(d-1) / 2} \operatorname{Re}\left(\delta_{1} \psi^{+} \bar{\psi}_{1}\right)+|x|^{(d-1) / 2}\left|\delta_{1} \psi^{+}\right|^{2}= \\
& \delta_{1,1} a+\delta_{1,2} a+\delta_{1,3} a
\end{aligned}
$$

where $\varphi=|k||x|-k x, f_{1}=f_{1}(k,|k| x /|x|)$ is the function of (1.8),

$$
\begin{aligned}
& \psi_{1}^{+}=e^{i k x}+\frac{e^{i|k||x|}}{|x|^{(d-1) / 2}} f_{1}\left(k,|k| \frac{x}{|x|}\right), \\
& \delta_{1} \psi^{+}=\psi^{+}-\psi_{1}^{+},
\end{aligned}
$$

where $\psi^{+}$is the function of (1.4), (1.8). 
Multipoint formulas for phase recovering

In addition, we have that (see [N2]):

$$
\begin{aligned}
& \left|\delta_{1} \psi^{+}(x, k)\right| \leq|x|^{-(d+1) / 2} C_{1}, \\
& \left|\delta_{1,1} a(x, k)\right| \leq|x|^{-(d-1) / 2}\left(F_{1}\right)^{2}, \\
& \left|\delta_{1,2} a(x, k)\right| \leq 2|x|^{-1} C_{1}\left(1+|x|^{-(d-1) / 2} F_{1}\right), \\
& \left|\delta_{1,3} a(x, k)\right| \leq|x|^{-(d+3) / 2}\left(C_{1}\right)^{2}, \\
& x \in \mathbb{R}^{d} \backslash\{0\}, \quad k \in \mathbb{R}^{d}, \quad k^{2}=E>0,
\end{aligned}
$$

where $C_{1}=C_{1}(v, E), \quad F_{1}=F_{1}(v, E)$ are positive constants depending on $v$ and $E$ only, in addition $F_{1}=\left\|f_{1}\right\|_{L^{\infty}\left(\Omega_{E}\right)}$.

Formulas (2.12)-(2.18) can be used for obtaining detailed versions of estimates (2.7), (2.8).

Note also that formulas $(2.2),(2.3)$ for $f_{1}$ can be rewritten as follows:

$$
\begin{aligned}
& \left(\begin{array}{c}
f_{1}(k, l) \\
\bar{f}_{1}(k, l)
\end{array}\right)=M\left(\left(\begin{array}{c}
a\left(x_{1}, k\right) \\
a\left(x_{2}, k\right)
\end{array}\right)-\left(\begin{array}{c}
\delta_{1} a\left(x_{1}, k\right) \\
\delta_{1} a\left(x_{2}, k\right)
\end{array}\right)\right), \\
& M=\frac{-1}{2 i \sin \left(\varphi_{2}-\varphi_{1}\right)}\left(\begin{array}{cc}
e^{-i \varphi_{2}} & -e^{-i \varphi_{1}} \\
-e^{i \varphi_{2}} & e^{i \varphi_{1}}
\end{array}\right) .
\end{aligned}
$$

Finally, note that formulas (1.8), (1.9), (2.2)-(2.8) also yield formulas (1.16) for $n=1$, $d \geq 3$ and formulas of the type (1.16) but with less precise $O\left(s^{-1}\right)$ in place of $O\left(s^{-3 / 2}\right)$ for $n=1, d=2$.

3. General idea of $\mathbf{2 n - p o i n t ~ f o r m u l a s ~ f o r ~} f$ and $\psi^{+}$for $d \geq 2$

The main drawback of the two-point formulas (1.9), (2.2)-(2.9) for finding phased $f$ from phaseless $\left|\psi^{+}\right|^{2}$ is a slow decay of the error as $s \rightarrow+\infty$; see (2.2), (2.7), (2.8). This drawback motivates our considerations given below.

For $d \geq 2$, to obtain rapidly convergent formulas for finding $f(k, l)$ from $\left|\psi^{+}(x, k)\right|^{2}$ at appropriate $2 \mathrm{n}$ points

$$
x=x_{1}(s), \ldots, x_{2 n}(s),
$$

where

$$
\begin{aligned}
& x_{i}(s)=r_{i}(s) \hat{l}, \quad i=1, \ldots, 2 n, \quad \hat{l}=l /|l|, \\
& r_{i_{1}}(s)<r_{i_{2}}(s) \text { for } i_{1}<i_{2}, \quad r_{1}(s)=s \rightarrow+\infty,
\end{aligned}
$$

we suggest the following general scheme.

We use that, under our assumptions (1.2), (1.7), formula (1.8) admits the following much more precise version:

$$
\psi^{+}(x, k)=e^{i k x}+\frac{e^{i|k||x|}}{|x|^{(d-1) / 2}}\left(\sum_{j=1}^{n} \frac{f_{j}\left(k,|k| \frac{x}{|x|}\right)}{|x|^{j-1}}+O\left(\frac{1}{|x|^{n}}\right)\right) \text { as }|x| \rightarrow \infty,
$$

where $x \in \mathbb{R}^{d}, k \in \mathbb{R}^{d}, k^{2}=E>0, n \in \mathbb{N}$. Actually, expansion (3.3) follows from assumptions (1.2), equation (1.4) and an extended version of Proposition 1.1 of [M]. Note also that formulas (1.9)-(1.11) for $f_{1}$ have analogs for $f_{j}, j \geq 2$. 
R.G. Novikov

\section{We look for}

formulas for finding $f_{j}(k, l)$ up to $O\left(s^{-(n-j+1)}\right)$ as $s \rightarrow+\infty, j=1, \ldots, n$,

from $\left|\psi^{+}(x, k)\right|^{2}$ given at $2 n$ points $x=x_{1}(s), \ldots, x_{2 n}(s)$ of the form (3.2),

where $f_{j}=f_{j}(k, l), j=1, \ldots, n$, are the functions arising in (3.3), $k$ and $l$ are fixed, $k, l \in \mathbb{R}^{d},|k|=|l|>0, l \neq k$.

In particular, in view of (1.9) and (3.4) for $j=1$ this scheme yields 2 n-point formulas for finding $f=f(k, l)$ from $\left|\psi^{+}\right|^{2}=\left|\psi^{+}(x, k)\right|^{2}$ given at $x=x_{1}(s), \ldots, x_{2 n}(s)$ of the form (3.2) with the error $O\left(s^{-n}\right)$ as $s \rightarrow+\infty$, that is with rapidly decaying error if $n$ is sufficiently large.

Besides, in view of (3.3), (3.4) this scheme also yields

formulas for finding $\psi^{+}(s \hat{l}, k)$ up to $O\left(s^{-n-(d-1) / 2}\right)$ as $s \rightarrow+\infty$

from $\left|\psi^{+}(x, k)\right|^{2}$ given at $2 n$ points $x=x_{1}(s), \ldots, x_{2 n}(s)$ of the form (3.2),

where $k$ and $l$ are fixed, $k, l \in \mathbb{R}^{d},|k|=|l|>0, l \neq k, \hat{l}=l /|l|$.

The realization of the general scheme of this Section depends on the concrete choice of the functions $r_{i}=r_{i}(s), i=1, \ldots, 2 n$, of (3.1), (3.2). In the present work we realize this scheme for the case when $r_{i}=r_{i}(s)$ are defined as in (1.15) for $d=3$ and for $d=2$. In particular, in this case $x_{2 j}(s)-x_{2 j-1}(s)=\tau \hat{l}, j=1, \ldots, n$, as well as $x_{2}(s)-x_{1}(s)=\tau \hat{l}$ for $x_{2}, x_{1}$ of the 2-point case considered in Section 2 (see formula (2.4)).

Note that, originally, the realization of the general scheme of this Section was started in $\left[\mathrm{NG}\right.$ ] for the case when $r_{i}(s)=\lambda_{i} s, i=1, \ldots, 2 n, 1=\lambda_{1}<\lambda_{2}, \ldots,<\lambda_{2 n}$.

Finally, our results on formulas $(1.14),(1.16),(3.4),(3.5)$, for $x_{1}(s), \ldots, x_{2 n}(s)$ defined by (1.15), can be summarized as follows.

For $d \geq 3, n=1$, and $x_{1}(s), x_{2}(s)$ defined according to (1.15) (or by (2.4)), formulas (1.14), (3.4) are realized (in [N2], [N4]) as formulas (2.2)-(2.9) of Section 2.

For $d=3, n=2$, and $x_{1}(s), \ldots, x_{4}(s)$ defined according to $(1.15)$ (or by $(6.1)$ ), formulas (1.14), (3.4) are realized as formulas (6.13)-(6.16), (6.5), (6.3) of Section 6.

For $d=3, n>2$, and $x_{1}(s), \ldots, x_{2 n}(s)$ defined by (1.15) (or by (7.1)), formulas (1.14), (3.4) are realized as inductive formulas (7.4), (7.5), (7.16),(7.12), (7.6)-(7.10) of Section 7.

For $d=2, n=1$, and $x_{1}(s), x_{2}(s)$ defined according to (1.15) (or by (2.4)), formulas (1.14), (3.4) with $O\left(s^{-1}\right)$ (and not with $O\left(s^{-1 / 2}\right)$ as in (2.2)-(2.9) for $d=2$ ) are realized as formulas (9.3), (9.4), (9.2) of Section 9 .

For $d=2, n=2$, and $x_{1}(s), \ldots, x_{4}(s)$ defined according to (1.15), formulas (1.14), (3.4) are realized as formulas (10.20)-(10.23), (10.13), (10.10), (10.11), (10.8), (10.9) of Section 10.

For $d=2, n>2$, and $x_{1}(s), \ldots, x_{2 n}(s)$ defined by (1.15), formulas (1.14), (3.4) are realized as inductive formulas (11.2), (11.6), (11.7) and (7.4) for $d=2,(11.11),(11.12)$ of Section 11.

Formulas (1.16), (3.5) are realized as formulas (12.1), (12.2) of Section 12.

Finally, note that detailed versions of $O\left(s^{-\sigma}\right)$ in $(2.7), O\left(s^{-(n-j+1)}\right)$ in (3.4) and $O\left(s^{-n-(d-1) / 2}\right)$ in $(3.5)$ will be given in subsequent papers. 
Multipoint formulas for phase recovering

4. Presentations for $a$ for $d=3$

The function $a$ defined by (2.1) can be presented as follows for $d=3$ :

$$
\begin{aligned}
& a(x, k)=a_{n}(x, k)+\delta_{n} a(x, k), \\
& a_{n}(x, k)=a_{n}^{1}(x, k)+a_{n}^{2}(x, k), \\
& a_{n}^{1}(x, k)=\sum_{j=1}^{n} \frac{e^{i \varphi} f_{j}}{|x|^{j-1}}+\sum_{j=1}^{n} \frac{e^{-i \varphi} \bar{f}_{j}}{|x|^{j-1}}, \\
& a_{n}^{2}(x, k)=\sum_{\substack{j \in \mathbb{N} \\
2 j \leq n}} \frac{\left|f_{j}\right|^{2}}{|x|^{2 j-1}}+\sum_{\substack{j_{1}, j_{2} \in \mathbb{N} \\
j_{1} \neq j_{2}, j_{1}+j_{2} \leq n}} \frac{f_{j_{1}} \bar{f}_{j_{2}}}{|x|^{j_{1}+j_{2}-1}}, \\
& \delta_{n} a(x, k)=O\left(\frac{1}{|x|^{n}}\right) \text { as }|x| \rightarrow \infty,
\end{aligned}
$$

where

$$
\begin{aligned}
& \varphi=\varphi(x, k)=|x|(|k|-k \hat{x}), \\
& f_{j}=f_{j}(k,|k| \hat{x}), \quad j=1, \ldots, n,
\end{aligned}
$$

$x \in \mathbb{R}^{3} \backslash\{0\}, \hat{x}=x /|x|, \quad k \in \mathbb{R}^{3}, k^{2}=E>0, \quad n \in \mathbb{N}$.

Formulas (4.1)-(4.7) follow from (2.1), (3.3) for $d=3$.

In addition, we have that:

$$
\begin{gathered}
a(x+\tau \hat{x}, k)=a_{n}^{1, \tau}(x, k)+\left(a_{n}^{1}(x+\tau \hat{x}, k)-a_{n}^{1, \tau}(x, k)\right)+a_{n}^{2}(x+\tau \hat{x}, k)+\delta_{n} a(x+\tau \hat{x}, k), \\
a_{n}^{1}(x+\tau \hat{x}, k)-a_{n}^{1, \tau}(x, k)=\Delta_{n}^{1, \tau}(x, k)+\rho_{n}^{1, \tau}(x, k),
\end{gathered}
$$

where

$$
\begin{aligned}
& a_{n}^{1, \tau}(x, k)=\sum_{j=1}^{n} \frac{e^{i \varphi_{\tau}} f_{j}}{|x|^{j-1}}+\sum_{j=1}^{n} \frac{e^{-i \varphi_{\tau}} \bar{f}_{j}}{|x|^{j-1}}, \\
& \Delta_{n}^{1, \tau}(x, k)=\sum_{j=1}^{n-1} \frac{e^{i \varphi_{\tau}} f_{j}}{|x|^{j-1}} w_{j-1}(|x|, \tau)+\sum_{j=1}^{n-1} \frac{e^{-i \varphi_{\tau}} \bar{f}_{j}}{|x|^{j-1}} w_{j-1}(|x|, \tau), \\
& \rho_{n}^{1, \tau}(x, k)=\frac{e^{i \varphi_{\tau}} f_{n}}{|x|^{n-1}} w_{n-1}(|x|, \tau)+\frac{e^{-i \varphi_{\tau}} \bar{f}_{n}}{|x|^{n-1}} w_{n-1}(|x|, \tau), \\
& w_{j-1}(|x|, \tau)=\frac{1}{(1+\tau /|x|)^{j-1}}-1=O\left(\frac{1}{|x|}\right),|x| \rightarrow \infty, \\
& \text { at fixed } \tau, j=1, \ldots, n \text {, } \\
& a_{n}^{2}(x+\tau \hat{x}, k)=\sum_{\substack{j \in \mathbb{N} \\
2 j \leq n}} \frac{\left|f_{j}\right|^{2}}{(|x|+\tau)^{2 j-1}}+\sum_{\substack{j_{1}, j_{2} \in \mathbb{N} \\
j_{1} \neq j_{2}, j_{1}+j_{2} \leq n}} \frac{f_{j_{1}} \bar{f}_{j_{2}}}{(|x|+\tau)^{j_{1}+j_{2}-1}}, \\
& \rho_{n}^{1, \tau}(x, k)=O\left(\frac{1}{|x|^{n}}\right), \quad \delta_{n} a(x+\tau \hat{x}, k)=O\left(\frac{1}{|x|^{n}}\right) \quad \text { as } \quad|x| \rightarrow \infty,
\end{aligned}
$$


R.G. Novikov

where

$$
\varphi_{\tau}=\varphi_{\tau}(x, k)=(|x|+\tau)(|k|-k \hat{x}),
$$

$f_{j}$ are the same that in (3.3), (4.3), (4.4), $x \in \mathbb{R}^{3} \backslash\{0\}, \hat{x}=x /|x|, \quad k \in \mathbb{R}^{3}, \quad k^{2}=E>0$, $n \in \mathbb{N}$.

\section{An advanced version of formulas (2.2)-(2.9) for $d=3$}

Let

$$
z_{n}(k, l, s)=\sum_{j=1}^{n} \frac{f_{j}(k, l)}{s^{j-1}}, \quad(k, l) \in \Omega_{E}, \quad s>0, \quad n \in \mathbb{N},
$$

where $f_{j}$ are the coefficients of (3.3).

Proposition 5.1. Under assumptions (1.2), (1.7), the following formulas hold, for $d=3$ :

$$
\begin{gathered}
\left(\begin{array}{c}
z_{n}(k, l, s) \\
\bar{z}_{n}(k, l, s)
\end{array}\right)=M\left(\begin{array}{c}
a(s \hat{l}, k) \\
a((s+\tau) \hat{l}, k)
\end{array}\right)- \\
M\left(\left(\begin{array}{c}
a_{n}^{2}(s \hat{l}, k) \\
\Delta_{n}^{1, \tau}(s \hat{l}, k)+a_{n}^{2}((s+\tau) \hat{l}, k)
\end{array}\right)+\left(\begin{array}{c}
\delta_{n} a(s \hat{l}, k) \\
\rho_{n}^{1, \tau}(s \hat{l}, k)+\delta_{n} a((s+\tau) \hat{l}, k)
\end{array}\right)\right), \\
M=M_{k, l, \tau, s}=\frac{-1}{2 i \sin \left(\varphi_{2}-\varphi_{1}\right)}\left(\begin{array}{cc}
e^{-i \varphi_{2}} & -e^{-i \varphi_{1}} \\
-e^{i \varphi_{2}} & e^{i \varphi_{1}}
\end{array}\right), \\
\varphi_{1}=(|k|-k \hat{l}) s, \quad \varphi_{2}=(|k|-k \hat{l})(s+\tau), \\
\delta_{n} a(s \hat{l}, k)=O\left(s^{-n}\right), \quad \delta_{n} a((s+\tau) \hat{l}, k)=O\left(s^{-n}\right) \text { as } s \rightarrow+\infty
\end{gathered}
$$

uniformly in $\hat{k}, \hat{l}$ and $\tau$ at fixed $E>0$,

$$
\rho_{n}^{1, \tau}(s \hat{l}, k)=O\left(s^{-n}\right) \text { as } s \rightarrow+\infty
$$

uniformly in $\hat{k}, \hat{l}$ at fixed $\tau>0$ and $E>0$, where

$$
\sin \left(\varphi_{2}-\varphi_{1}\right) \neq 0
$$

$k \in \mathbb{R}^{3}, \quad l \in \mathbb{R}^{3}, \quad|k|=|l|=\sqrt{E}, \hat{k}=k /|k|, \hat{l}=l /|l|, \quad \tau>0, \quad n \in \mathbb{N}$.

Proof of Proposition 5.1. Using (4.1)-(4.3), (4.8)-(4.10), (5.1) we obtain that

$$
\begin{aligned}
& a(s \hat{l}, k)=e^{i \varphi_{1}} z_{n}(k, l, s)+e^{-i \varphi_{1}} \bar{z}_{n}(k, l, s)+a_{n}^{2}(s \hat{l}, k)+\delta_{n} a(s \hat{l}, k), \\
& a((s+\tau) \hat{l}, k)=e^{i \varphi_{2}} z_{n}(k, l, s)+e^{-i \varphi_{2}} \bar{z}_{n}(k, l, s)+ \\
& \Delta_{n}^{1, \tau}(s \hat{l}, k)+a_{n}^{2}((s+\tau) \hat{l}, k)+\rho_{n}^{1, \tau}(s \hat{l}, k)+\delta_{n} a((s+\tau) \hat{l}, k),
\end{aligned}
$$

where $\varphi_{1}, \varphi_{2}$ are given by (5.4).

Considering (5.8), (5.9) as a linear system for $z_{n}, \bar{z}_{n}$ we obtain (5.2), (5.3). 
Multipoint formulas for phase recovering

Estimates (5.5), (5.6) follow from (4.5), (4.12), (4.13).

Proposition 5.1 is proved.

Actually, formulas (5.2)-(5.7) for $n=1$ reduce to formulas (2.2)-(2.9) (with (2.2), (2.3) written as (2.19), (2.20)), $d=3$.

\section{Finding $f_{1}, f_{2}$ for the 4-point case for $d=3$}

In this section we realize the scheme of Section 3 for the case when $n=2, d=3$ and

$$
x_{1}=s \hat{l}, x_{2}=(s+\tau) \hat{l}, x_{3}=\lambda s \hat{l}, x_{4}=(\lambda s+\tau) \hat{l},
$$

where $\tau>0$ and $\lambda>1$ are fixed, $s \rightarrow+\infty$.

6.1. Considerations at $x_{1}$ and $x_{2}$. Due to (5.2)-(5.7) for $n=1$ (or, by other words, due to $(2.19),(2.20),(2.4)-(2.9)$ for $d=3)$, we have that

$$
\begin{aligned}
& f_{1}=f_{1,1}(s)+O\left(s^{-1}\right) \text { as } s \rightarrow+\infty \\
& \text { for fixed } \sin ((|k|-k \hat{l}) \tau) \neq 0 \text { and } E,
\end{aligned}
$$

where $f_{1}=f_{1}(k, l), f_{1,1}(s)=f_{1,1}(k, l, \tau, s)$,

$$
\left(\begin{array}{c}
f_{1,1}(s) \\
\bar{f}_{1,1}(s)
\end{array}\right)=M\left(\begin{array}{c}
a\left(x_{1}, k\right) \\
a\left(x_{2}, k\right)
\end{array}\right)
$$

$M=M_{k, l, \tau, s}$ is defined by (5.3), $x_{1}, x_{2}$ are defined in $(6.1),(k, l) \in \Omega_{E}, d=3, E>0$, $\hat{l}=l /|l|, \tau>0$.

Proposition 6.1. Under assumptions (1.2), (1.7) for $d=3$, the following formulas hold:

$$
\begin{aligned}
& z_{2}(s)=z_{2,1}(s)+O\left(s^{-2}\right) \text { as } s \rightarrow+\infty \\
& \text { for fixed } \sin ((|k|-k \hat{l}) \tau) \neq 0, E \text { and } \tau,
\end{aligned}
$$

where $z_{2}(s)=z_{2}(k, l, s)$ is defined by (5.1) for $n=2, z_{2,1}(s)=z_{2,1}(k, l, \tau, s)$ is defined as follows:

$$
\left(\begin{array}{c}
z_{2,1}(s) \\
\bar{z}_{2,1}(s)
\end{array}\right)=\left(\begin{array}{c}
f_{1,1}(s) \\
\bar{f}_{1,1}(s)
\end{array}\right)-\frac{\left|f_{1,1}(s)\right|^{2}}{s} M\left(\begin{array}{l}
1 \\
1
\end{array}\right),
$$

$f_{1,1}(s)=f_{1,1}(k, l, \tau, s)$ is defined by (6.3), $M=M_{k, l, \tau, s}$ is defined by (5.3), $(k, l) \in \Omega_{E}$, $d=3, E>0, \hat{l}=l /|l|, \tau>0$.

Proof of Proposition 6.1. Proposition 6.1 follows from Proposition 5.1 for $n=2$ and from formulas (6.2), (6.3). In more detail, the proof consists of the following.

Formulas (4.4), (4.11), (4.13), (4.14) for $n=2$ imply that

$$
\begin{aligned}
& a_{2}^{2}(s \hat{l}, k)=\frac{\left|f_{1}(k, l)\right|^{2}}{s} \\
& \Delta_{2}^{1, \tau}(s \hat{l}, k)=0 \\
& a_{2}^{2}((s+\tau) \hat{l}, k)=\frac{\left|f_{1}(k, l)\right|^{2}}{s+\tau},
\end{aligned}
$$


R.G. Novikov

$s>0, \tau>0$.

Formulas (5.2)-(5.7) for $n=2$ and formulas (6.6)-(6.8) imply that

$$
\left(\begin{array}{c}
z_{2}(s) \\
\bar{z}_{2}(s)
\end{array}\right)=M\left(\begin{array}{c}
a(s \hat{l}, k) \\
a((s+\tau) \hat{l}, k)
\end{array}\right)-M\left(\begin{array}{c}
s^{-1}\left|f_{1}\right|^{2} \\
(s+\tau)^{-1}\left|f_{1}\right|^{2}
\end{array}\right)+O\left(\frac{1}{s^{2}}\right) \text { as } s \rightarrow+\infty
$$

for fixed $\sin ((|k|-k \hat{l}) \tau) \neq 0, E$ and $\tau$.

Formulas (6.2), (6.3), (6.9) imply (6.4), (6.5).

This completes the proof of Proposition 6.1.

6.2. Considerations at $x_{3}$ and $x_{4}$. In addition to (6.4), we have that

$$
\begin{aligned}
& z_{2}(\lambda s)=z_{2,1}(\lambda s)+O\left((\lambda s)^{-2}\right) \text { as } s \rightarrow+\infty \\
& \text { for fixed } \sin ((|k|-k \hat{l}) \tau) \neq 0, E \text { and } \tau .
\end{aligned}
$$

The point is that $z_{2,1}(\lambda s)$ is given in terms of $a\left(x_{3}, k\right), a\left(x_{4}, k\right)$ via formulas (6.3), (6.5) with $x_{3}, x_{4}$ in place of $x_{1}, x_{2}$ and $\lambda s$ in place of $s$, where $x_{1}, x_{2}, x_{3}, x_{4}$ are defined by (6.1).

6.3. Formulas for $f_{1}, f_{2}$. Due to definition (5.1), for $n=2$, and formulas (6.4), (6.10), we have that

$$
\begin{aligned}
& f_{1}+s^{-1} f_{2}=z_{2,1}(s)+O\left(s^{-2}\right), \quad s \rightarrow+\infty, \\
& f_{1}+(\lambda s)^{-1} f_{2}=z_{2,1}(\lambda s)+O\left((\lambda s)^{-2}\right), \quad s \rightarrow+\infty, \\
& \text { for fixed } \sin ((|k|-k \hat{l}) \tau) \neq 0, E \text { and } \tau,
\end{aligned}
$$

where $f_{1}=f_{1}(k, l), f_{2}=f_{2}(k, l)$,

$z_{2,1}(s)=z_{2,1}(k, l, \tau, s),(k, l) \in \Omega_{E}, d=3, E>0, \hat{l}=l /|l|, \tau>0$.

Proceeding from $(6.11),(6.12)$ we obtain the following result.

Proposition 6.2. Under assumptions (1.2), (1.7) for $d=3$, the following formulas hold:

$$
\begin{aligned}
& f_{1}=f_{1,2}(s)+O\left(s^{-2}\right), \quad s \rightarrow+\infty, \\
& f_{2}=f_{2,1}(s)+O\left(s^{-1}\right), \quad s \rightarrow+\infty, \\
& \text { for fixed } \sin ((|k|-k \hat{l}) \tau) \neq 0, E, \tau \text { and } \lambda,
\end{aligned}
$$

where $f_{1}=f_{1}(k, l), f_{2}=f_{2}(k, l)$,

$f_{1,2}(s)=f_{1,2}(k, l, \tau, \lambda, s), \quad f_{2,1}(s)=f_{2,1}(k, l, \tau, \lambda, s)$,

$$
\begin{aligned}
& f_{1,2}(s)=-\frac{z_{2,1}(s)}{\lambda-1}+\frac{\lambda z_{2,1}(\lambda s)}{\lambda-1} \\
& f_{2,1}(s)=\frac{s \lambda}{\lambda-1}\left(z_{2,1}(s)-z_{2,1}(\lambda s)\right),
\end{aligned}
$$


Multipoint formulas for phase recovering

where $z_{2,1}(s)=z_{2,1}(k, l, \tau, s)$ is found via (6.3), (6.5) from $\left|\psi^{+}(x, k)\right|^{2}$ at $x=x_{1}, x_{2}$ of (6.1), $z_{2,1}(\lambda s)=z_{2,1}(k, l, \tau, \lambda s)$ is found via (6.3), (6.5) (where $s$ and $x_{1}, x_{2}$ are replaced by $\lambda s$ and $x_{3}, x_{4}$ of $\left.(6.1)\right),(k, l) \in \Omega_{E}, d=3, E>0, \hat{l}=l /|l|, \tau>0, \lambda>1$.

Propositions 6.1 and 6.2 realize the scheme of Section 3 , for $n=2, d=3$, for finding $f_{1}, f_{2}$ as in (3.3) from $\left|\psi^{+}(x, k)\right|^{2}$ given at $x=x_{1}, x_{2}, x_{3}, x_{4}$ defined in (6.1).

7. Inductive finding $f_{1}, \ldots, f_{n}$ for the $2 n$-point case for $d=3$

In this section we realize the scheme of Section 3 for the case when $d=3$ and $x_{1}, \ldots, x_{2 n}$ are defined as in (1.15).

\subsection{Step of induction. Suppose that}

$$
\begin{aligned}
& f_{1, n-1}(s), f_{2, n-2}(s), \ldots, f_{n-1,1}(s) \text { are functions found from } \\
& \left|\psi^{+}(x, k)\right|^{2} \text { given at } x=x_{1}(s), \ldots, x_{2(n-1)}(s) \text { defined in }
\end{aligned}
$$

and such that

$$
\begin{aligned}
& f_{j}=f_{j, n-j}(s)+O\left(s^{-(n-j)}\right), \quad s \rightarrow+\infty, \quad j=1, \ldots, n-1, \\
& \text { for fixed } \sin ((|k|-k \hat{l}) \tau) \neq 0, E, \tau \text { and } \lambda_{1}, \ldots, \lambda_{n-1},
\end{aligned}
$$

where $f_{j}=f_{j}(k, l), f_{j, n-j}(s)=f_{j, n-j}\left(k, l, \tau, \lambda_{1}, \ldots, \lambda_{n-1}, s\right),(k, l) \in \Omega_{E}, d=3, E>0$, $\hat{l}=l /|l|, \tau>0$. For $n=2$ and $n=3$ such functions are found in Sections 2 and 6 .

The step of induction consists in finding $f_{1, n}, f_{2, n-1}, \ldots, f_{n, 1}$ proceeding from $f_{1, n-1}, f_{2, n-2}, \ldots, f_{n-1,1}$ of $(7.1),(7.2)$, where

$$
\begin{aligned}
& f_{1, n}(s), f_{2, n-1}(s), \ldots, f_{n, 1}(s) \text { are functions found from } \\
& \left|\psi^{+}(x, k)\right|^{2} \text { given at } x=x_{1}(s), \ldots, x_{2 n}(s) \text { defined by }
\end{aligned}
$$

and such that

$$
\begin{aligned}
& f_{j}=f_{j, n-j+1}(s)+O\left(s^{-(n-j+1)}\right), \quad s \rightarrow+\infty, \quad j=1, \ldots, n, \\
& \text { for fixed } \sin ((|k|-k \hat{l}) \tau) \neq 0, E, \quad \tau \text { and } \lambda_{1}, \ldots, \lambda_{n},
\end{aligned}
$$

where $f_{j}=f_{j}(k, l), f_{j, n-j+1}(s)=f_{j, n-j+1}\left(k, l, \tau, \lambda_{1}, \ldots, \lambda_{n}, s\right),(k, l) \in \Omega_{E}, d=3, E>0$, $\hat{l}=l /|l|, \tau>0$.

This step of induction is realized via Theorems 7.1 and 7.2 formulated in the next subsection.

7.2. Realization of the inductive step. Let $f_{1,1}(\kappa s)=f_{1,1}(k, l, \tau, \kappa s)$ be defined by

$$
\left(\begin{array}{c}
f_{1,1}(\kappa s) \\
\bar{f}_{1,1}(\kappa s)
\end{array}\right)=M\left(\begin{array}{c}
a\left(y_{1}, k\right) \\
a\left(y_{2}, k\right)
\end{array}\right)
$$

where $M=M_{k, l, \tau, \kappa s}$ is defined according to (5.3), (5.4), (5.7), a is defined by (2.1) (for $d=3)$,

$$
y_{1}=\kappa s \hat{l}, \quad y_{2}=(\kappa s+\tau) \hat{l},
$$


R.G. Novikov

$(k, l) \in \Omega_{E}, d=3, E>0, \hat{l}=l /|l|, s>0, \kappa \geq 1$.

Let

$$
\begin{aligned}
& a_{n, n-1}^{2}(\kappa, s)=\sum_{\substack{j \in \mathbb{N} \\
2 j \leq n}} \frac{\left|f_{j, n-j}(s)\right|^{2}}{(\kappa s)^{2 j-1}}+\sum_{\substack{j_{1}, j_{2} \in \mathbb{N} \\
j_{1} \neq j_{2}, j_{1}+j_{2} \leq n}} \frac{f_{j_{1}, n-j_{1}}(s) \bar{f}_{j_{2}, n-j_{2}}(s)}{(\kappa s)^{j_{1}+j_{2}-1}} \\
& a_{n, n-1}^{2, \tau}(\kappa, s)=\sum_{\substack{j \in \mathbb{N} \\
2 j \leq n}} \frac{\left|f_{j, n-j}(s)\right|^{2}}{(\kappa s+\tau)^{2 j-1}}+\sum_{\substack{j_{1}, j_{2} \in \mathbb{N} \\
j_{1} \neq j_{2}, j_{1}+j_{2} \leq n}} \frac{f_{j_{1}, n-j_{1}}(s) \bar{f}_{j_{2}, n-j_{2}}(s)}{(\kappa s+\tau)^{j_{1}+j_{2}-1}}, \\
& \Delta_{n, n-1}^{1, \tau}(\kappa, s)=\sum_{j=1}^{n-1} \frac{e^{i \varphi_{\tau}(\kappa s)} f_{j, n-j}(s)}{(\kappa s)^{j-1}} w_{j-1}(\kappa s, \tau)+ \\
& \sum_{j=1}^{n-1} \frac{e^{-i \varphi_{\tau}(\kappa s)} \bar{f}_{j, n-j}(s)}{(\kappa s)^{j-1}} w_{j-1}(\kappa s, \tau),
\end{aligned}
$$

where $f_{j, n-j}(s)$ and $w_{j-1}(s, \tau), j=1, \ldots, n-1$, are the functions of (7.1), (7.2) and (4.13), $\varphi_{\tau}(s)=\varphi_{\tau}(s \hat{l}, k)$, where $\varphi_{\tau}(x, k)$ is defined by $(4.16), \hat{l}=l /|l|$.

Theorem 7.1. Under assumptions (1.2), (1.7) for $d=3$, the following formulas hold:

$$
\begin{aligned}
& z_{n}(\kappa s)=\zeta_{n, n-1}(\kappa, s)+O\left(s^{-n}\right) \text { as } s \rightarrow+\infty \\
& \text { for fixed } \sin ((|k|-k \hat{l}) \tau) \neq 0, E, \tau \text { and } \lambda_{1}, \ldots, \lambda_{n-1}, \kappa,
\end{aligned}
$$

where $z_{n}(s)=z_{n}(k, l, s)$ is defined by $(5.1)$,

$\zeta_{n, n-1}(\kappa, s)=\zeta_{n, n-1}\left(k, l, \tau, \lambda_{1}, \ldots, \lambda_{n-1}, \kappa, s\right)$ is defined as follows:

$$
\left(\begin{array}{c}
\zeta_{n, n-1}(\kappa, s) \\
\bar{\zeta}_{n, n-1}(\kappa, s)
\end{array}\right)=\left(\begin{array}{c}
f_{1,1}(\kappa s) \\
\bar{f}_{1,1}(\kappa s)
\end{array}\right)-M\left(\begin{array}{c}
a_{n, n-1}^{2}(\kappa, s) \\
\Delta_{n, n-1}^{1, \tau}(\kappa, s)+a_{n, n-1}^{2, \tau}(\kappa, s)
\end{array}\right)
$$

$f_{1,1}(\kappa s)$ is defined by (7.5), $M=M_{k, l, \tau, \kappa s}$ is defined according to (5.3), (5.4), $a_{n, n-1}^{2}(\kappa, s)$, $a_{n, n-1}^{2, \tau}(\kappa, s)$ and $\Delta_{n, n-1}^{1, \tau}(\kappa, s)$ are defined by (7.7)-(7.9), $(k, l) \in \Omega_{E}, d=3, E>0, \hat{l}=l /|l|$, $\tau>0, \lambda_{1}, \ldots, \lambda_{n-1}$ are the numbers of (1.15), $\kappa \geq 1, n \geq 2$.

In Theorem 7.1 we have that:

(a) $\zeta_{n, n-1}(\kappa, s)$ appropriately approximates $z_{n}(\kappa s)$ as $s \rightarrow+\infty$;

(b) $\zeta_{n, n-1}(\kappa, s)$ is given in terms of $f_{1,1}(\kappa s)$ defined by $(7.5)$ and $f_{j, n-j}(s)$, $j=1, \ldots, n-1$, of $(7.1),(7.2)$ (in view of (7.11), (7.7)-(7.9)), and, as a corollary, $\zeta_{n, n-1}(\kappa, s)$ is found from $\left|\psi^{+}(x, k)\right|^{2}$ at $x=x_{1}(s), \ldots, x_{2(n-1)}(s), y_{1}(s), y_{2}(s)$ of (1.15) and (7.6).

Theorem 7.1 for $\kappa=1$ is an extension of Proposition 6.1 to the case $n>2$. In addition for $n=2$, in general, $z_{2,1}(\kappa s) \neq \zeta_{2,1}(\kappa, s)$.

Let

$$
\Lambda=\left(\Lambda_{j_{1}, j_{2}}\right)=\left(\lambda_{j_{1}}^{1-j_{2}}\right), \quad j_{1}, j_{2}=1, \ldots, n
$$


Multipoint formulas for phase recovering

where $\lambda_{1}, \ldots, \lambda_{n}$ are the numbers of (1.15). One can see that $\Lambda$ is a Vandermonde matrix and, in particular,

$$
\operatorname{det} \Lambda=\prod_{1 \leq i<j \leq n}\left(\lambda_{j}^{-1}-\lambda_{i}^{-1}\right) \neq 0
$$

where we used our assumptions on $\lambda_{j}$ of (1.15) in order to have that $\operatorname{det} \Lambda \neq 0$.

Theorem 7.2. Under assumptions (1.2), (1.7) for $d=3$, formulas (7.4) hold, where

$$
f_{i, n-i+1}(s)=s^{i-1} \sum_{j=1}^{n} \Lambda_{i, j}^{-1} \zeta_{n, n-1}\left(\lambda_{j}, s\right), \quad i=1, \ldots, n,
$$

where $\Lambda^{-1}=\left(\Lambda_{i, j}^{-1}\right)$ is the inverse of the matrix $\Lambda$ defined by $(7.12), \zeta_{n, n-1}(\kappa, s)$ is defined by $(7.11), n \geq 2$.

Note that $f_{1,2}, f_{2,1}$ of Theorem 7.2 for $n=2$ are different, in general, from $f_{1,2}, f_{2,1}$ of Proposition 6.2.

Theorems 7.1 and 7.2 realize the step of induction of Subsection 7.1.

\subsection{Proofs of Theorems 7.1 and 7.2.}

Proof of Theorem 7.1. Theorem 7.1 follows from Proposition 5.1, formula (7.5) and the formulas

$$
\begin{aligned}
& a_{n}^{2}(\kappa s)=a_{n, n-1}^{2}(\kappa, s)+O\left(s^{-n}\right), \quad s \rightarrow+\infty \\
& a_{n}^{2}(\kappa s+\tau)=a_{n, n-1}^{2, \tau}(\kappa, s)+O\left(s^{-n}\right), \quad s \rightarrow+\infty \\
& \Delta_{n}^{1, \tau}(\kappa s)=\Delta_{n, n-1}^{1, \tau}(\kappa, s)+O\left(s^{-n}\right), \quad s \rightarrow+\infty \\
& \text { for fixed } \sin ((|k|-k \hat{l}) \tau) \neq 0, E, \tau \text { and } \lambda_{1}, \ldots, \lambda_{n-1},
\end{aligned}
$$

where $a_{n}^{2}(\kappa s)=a_{n}^{2}(\kappa s \hat{l}, k), a_{n}^{2}(\kappa s+\tau)=a_{n}^{2}((\kappa s+\tau) \hat{l}, k), \Delta_{n}^{1, \tau}(\kappa s)=\Delta_{n}^{1, \tau}(\kappa s \hat{l}, k)$ are the functions of (4.4), (4.14), (4.11), $a_{n, n-1}^{2}(\kappa, s), a_{n, n-1}^{2, \tau}(\kappa, s), \Delta_{n, n-1}^{1, \tau}(\kappa, s)$ are the functions of $(7.7),(7.8),(7.9)$.

Formula (7.15) follows from (4.4), (7.7), (7.2) and the observation that the terms

$$
\begin{aligned}
& O\left(s^{-(n+j-1)}\right), O\left(s^{-\left(n+j_{1}-1\right)}\right), O\left(s^{-\left(n+j_{2}-1\right)}\right), \\
& j \in \mathbb{N}, 2 j \leq n, j_{1}, j_{2} \in \mathbb{N}, j_{1} \neq j_{2}, j_{1}+j_{2} \leq n,
\end{aligned}
$$

arising at comparison of the summands of $a_{n}^{2}(\kappa s)$ and $a_{n, n-1}^{2}(\kappa, s)$ are majorized by $O\left(s^{-n}\right)$ as $s \rightarrow+\infty$.

Formula (7.16) is proved in a similar way with formula (7.15).

Formula (7.17) follows from(4.11), (4.13), (7.9), (7.2).

This completes the proof of Theorem 7.1.

Proof of Theorem 7.2. Formula (5.1) and formula (7.10) for $\lambda=\lambda_{1}, \ldots, \lambda_{n}$ imply the following system of approximate equations for $f_{1}, \ldots, f_{n}$ :

$$
\sum_{j=1}^{n} \frac{f_{j}}{\left(\lambda_{i} s\right)^{j-1}}=\zeta_{n, n-1}\left(\lambda_{i}, s\right)+O\left(s^{-n}\right) \text { as } s \rightarrow+\infty, \quad i=1, \ldots, n
$$


R.G. Novikov

where $\lambda_{1}, \ldots, \lambda_{n}$ are the number of (1.15).

Let

$$
\begin{aligned}
& g(s)=\left(g_{1}(s), \ldots, g_{n}(s)\right)^{T}, \quad g_{j}(s)=\frac{f_{j}}{s^{j-1}}, \quad j=1, \ldots, n, \\
& h(s)=\left(h_{1}(s), \ldots, h_{n}(s)\right)^{T}, \quad h_{j}(s)=\zeta_{n, n-1}\left(\lambda_{j}, s\right), j=1, \ldots, n .
\end{aligned}
$$

Then (7.18) can be rewritten as

$$
\Lambda g(s)=h(s)+O\left(s^{-n}\right) \text { as } s \rightarrow+\infty
$$

where $O\left(s^{-n}\right)$ is considered as an $n$-dimensional vector with the components $O\left(s^{-n}\right)$.

From (7.21) we obtain that

$$
g(s)=\Lambda^{-1} h(s)+O\left(s^{-n}\right) \text { as } s \rightarrow+\infty .
$$

Formulas (7.4), (7.14) follow from (7.19), (7.20), (7.22).

Theorem 7.2 is proved.

\section{Analogs for $d=2$ of formulas of Sections 4 and 5}

Formulas (4.1)-(4.16) of Section 4 and formulas (5.1)-(5.9) of Section 5 have direct analogs for $d=2$. The difference consists in the following modifications in formulas (4.4), (4.5), (4.14), (4.15) and (5.5):

$$
a_{n}^{2}(x, k)=\sum_{\substack{j \in \mathbb{N} \\ 2 j \leq n}} \frac{\left|f_{j}\right|^{2}}{|x|^{2 j-1-1 / 2}}+\sum_{\substack{j_{1}, j_{2} \in \mathbb{N} \\ j_{1} \neq j_{2}, j_{1}+j_{2} \leq n}} \frac{f_{j_{1}} \bar{f}_{j_{2}}}{|x|^{j_{1}+j_{2}-1-1 / 2}}
$$

(in place of (4.4));

$$
\delta_{n} a(x, k)=O\left(\frac{1}{|x|^{n-1 / 2}}\right) \quad \text { as } \quad|x| \rightarrow \infty
$$

(in place of $(4.5)$ );

$$
a_{n}^{2}(x+\tau \hat{x}, k)=\sum_{\substack{j \in \mathbb{N} \\ 2 j \leq n}} \frac{\left|f_{j}\right|^{2}}{(|x|+\tau)^{2 j-1-1 / 2}}+\sum_{\substack{j_{1}, j_{2} \in \mathbb{N} \\ j_{1} \neq j_{2}, j_{1}+j_{2} \leq n}} \frac{f_{j_{1}} \bar{f}_{j_{2}}}{(|x|+\tau)^{j_{1}+j_{2}-1-1 / 2}}
$$

(in place of (4.14));

$$
\delta_{n} a(x+\tau \hat{x}, k)=O\left(\frac{1}{|x|^{n-1 / 2}}\right) \text { as }|x| \rightarrow \infty
$$

(in place of the second term of formula (4.15));

$$
\delta_{n} a(s \hat{l}, k)=O\left(s^{-n+1 / 2}\right), \quad \delta_{n} a((s+\tau) \hat{l}, k)=O\left(s^{-n+1 / 2}\right) \quad \text { as } s \rightarrow+\infty
$$


Multipoint formulas for phase recovering

(in place of (5.5)).

Besides, for $d=2$, in all formulas of Sections 4 and 5 with modifications summed up in (8.1)-(8.5) we have that $x \in \mathbb{R}^{2} \backslash\{0\}, k \in \mathbb{R}^{2}, l \in \mathbb{R}^{2}$.

9. Improved finding $f_{1}$ for the 2-point case for $d=2$

Due to (2.19)-(2.20), (2.4)-(2.9) for $d=2$, we have that

$$
\begin{aligned}
& f_{1}=f_{1,1 / 2}(s)+O\left(s^{-1 / 2}\right) \text { as } s \rightarrow+\infty \\
& \text { for fixed } \sin ((|k|-k \hat{l}) \tau) \neq 0 \text { and } E,
\end{aligned}
$$

where $f_{1}=f_{1}(k, l), f_{1,1 / 2}(s)=f_{1,1 / 2}(k, l, \tau, s)$,

$$
\left(\begin{array}{c}
f_{1,1 / 2}(s) \\
\bar{f}_{1,1 / 2}(s)
\end{array}\right)=M\left(\begin{array}{c}
a\left(x_{1}, k\right) \\
a\left(x_{2}, k\right)
\end{array}\right)
$$

$M=M_{k, l, \tau, s}$ is defined by (5.3), $x_{1}, x_{2}$ are defined by $(2.4),(k, l) \in \Omega_{E}, d=2, E>0$, $\hat{l}=l /|l|, \tau>0$.

Proposition 9.1 Under assumptions (1.2), (1.7) for $d=2$, the following formulas hold:

$$
\begin{aligned}
& f_{1}=f_{1,1}(s)+O\left(s^{-1}\right) \text { as } s \rightarrow+\infty \\
& \text { for fixed } \sin ((|k|-k \hat{l}) \tau) \neq 0, E \text { and } \tau,
\end{aligned}
$$

where $f_{1,1}(s)=f_{1,1}(k, l, \tau, s)$ is defined as follows:

$$
\left(\begin{array}{c}
f_{1,1}(s) \\
\bar{f}_{1,1}(s)
\end{array}\right)=\left(\begin{array}{c}
f_{1,1 / 2}(s) \\
\bar{f}_{1,1 / 2}(s)
\end{array}\right)-\frac{\left|f_{1,1 / 2}(s)\right|^{2}}{s^{1 / 2}} M\left(\begin{array}{l}
1 \\
1
\end{array}\right)
$$

$f_{1,1 / 2}(s)=f_{1,1 / 2}(k, l, \tau, s)$ is defined by $(9.2), M=M_{k, l, \tau, s}$ is defined by $(5.3),(k, l) \in \Omega_{E}$, $d=2, E>0, \hat{l}=l /|l|, \tau>0$.

Proof of Proposition 9.1. Proposition 9.1 follows from Proposition 5.1 for $n=2, d=2$ and formulas (9.1), (9.2). In more detail, the proof consists of the following.

Formulas (8.1), (4.11), (4.13), (8.3) for $n=2$ imply that

$$
\begin{aligned}
& a_{2}^{2}(s \hat{l}, k)=\frac{\left|f_{1}(k, l)\right|^{2}}{s^{1 / 2}}, \\
& \Delta_{2}^{1, \tau}(s \hat{l}, k)=0, \\
& a_{2}^{2}((s+\tau) \hat{l}, k)=\frac{\left|f_{1}(k, l)\right|^{2}}{(s+\tau)^{1 / 2}},
\end{aligned}
$$

$s>0, \tau>0$.

Formulas (5.2)-(5.4), (8.5), (5.6), (5.7) for $n=2$ and formulas (9.5)-(9.7) imply that

$$
\begin{aligned}
& \left(\begin{array}{c}
z_{2}(s) \\
\bar{z}_{2}(s)
\end{array}\right)=M\left(\begin{array}{c}
a(s \hat{l}, k) \\
a((s+\tau) \hat{l}, k)
\end{array}\right)-M\left(\begin{array}{c}
s^{-1 / 2}\left|f_{1}\right|^{2} \\
(s+\tau)^{-1 / 2}\left|f_{1}\right|^{2}
\end{array}\right)+ \\
& O\left(\frac{1}{s^{3 / 2}}\right) \text { as } s \rightarrow+\infty
\end{aligned}
$$

for fixed $\sin ((|k|-k \hat{l}) \tau) \neq 0, E$ and $\tau$. 
R.G. Novikov

Formula (5.1), for $n=2$, and formulas (9.1), (9.2), (9.8) imply (9.3), (9.4).

This completes the proof of Proposition 9.1.

10. Finding $f_{1}, f_{2}$ for the 4 -point case for $d=2$

In this section we realize the scheme of Section 3 for the case when $n=2, d=2$ and $x_{1}, x_{2}, x_{3}, x_{4}$ are defined as in (6.1).

Proceeding from considerations of Section 9 at $x_{1}$ and $x_{2}$, we obtain the following result.

Proposition 10.1 Under assumptions (1.2), (1.7) for $d=2$, the following formulas hold:

$$
\begin{aligned}
& z_{2}(s)=z_{2,1}(s)+O\left(s^{-3 / 2}\right) \text { as } s \rightarrow+\infty \\
& \text { for fixed } \sin ((|k|-k \hat{l}) \tau) \neq 0, E \text { and } \tau,
\end{aligned}
$$

where $z_{2}(s)=z_{2}(k, l, s)$ is defined by (5.1) for $n=2, z_{2,1}(s)=z_{2,1}(k, l, \tau, s)$ is defined as follows:

$$
\left(\begin{array}{c}
z_{2,1}(s) \\
\bar{z}_{2,1}(s)
\end{array}\right)=\left(\begin{array}{c}
f_{1,1 / 2}(s) \\
\bar{f}_{1,1 / 2}(s)
\end{array}\right)-\frac{\left|f_{1,1}(s)\right|^{2}}{s^{1 / 2}} M\left(\begin{array}{l}
1 \\
1
\end{array}\right),
$$

$f_{1,1 / 2}(s)=f_{1,1 / 2}(k, l, \tau, s)$ is defined by (9.2), $f_{1,1}(s)=f_{1,1}(k, l, \tau, s)$ is defined by (9.4), $M=M_{k, l, \tau, s}$ is defined by (5.3), $(k, l) \in \Omega_{E}, d=2, E>0, \hat{l}=l /|l|, \tau>0$.

Proposition 10.1 follows from formulas (9.2), (9.3), (9.8).

Due to similar considerations at $x_{3}$ and $x_{4}$, we also have that

$$
\begin{aligned}
& z_{2}(\lambda s)=z_{2,1}(\lambda s)+O\left((\lambda s)^{-3 / 2}\right) \text { as } s \rightarrow+\infty \\
& \text { for fixed } \sin ((|k|-k \hat{l}) \tau) \neq 0, E \text { and } \tau,
\end{aligned}
$$

where $z_{2,1}(\lambda s)$ is given in terms of $a\left(x_{3}, k\right), a\left(x_{4}, k\right)$ via formulas (9.2), (9.4), (10.2) with $\lambda s$ and $x_{3}, x_{4}$ in place of $s$ and $x_{1}, x_{2}$.

Next, due to definition (5.1), for $n=2$, and formulas (10.1), (10.3), we have that

$$
\begin{aligned}
& f_{1}+s^{-1} f_{2}=z_{2,1}(s)+O\left(s^{-3 / 2}\right) \text { as } s \rightarrow+\infty, \\
& f_{1}+(\lambda s)^{-1} f_{2}=z_{2,1}(\lambda s)+O\left((\lambda s)^{-3 / 2}\right) \text { as } s \rightarrow+\infty, \\
& \text { for fixed } \sin ((|k|-k \hat{l}) \tau) \neq 0, E \text { and } \tau,
\end{aligned}
$$

where $f_{1}=f_{1}(k, l), f_{2}=f_{2}(k, l), z_{2,1}(s)=z_{2,1}(k, l, \tau, s),(k, l) \in \Omega_{E}, d=2, E>0$, $\hat{l}=l /|l|, \tau>0$.

Proceeding from (10.4), (10.5), we obtain the following result.

Proposition 10.2. Under assumptions (1.2), (1.7) for $d=2$, the following formulas hold:

$$
\begin{aligned}
& f_{1}=f_{1,3 / 2}(s)+O\left(s^{-3 / 2}\right) \text { as } s \rightarrow+\infty, \\
& f_{2}=f_{2,1 / 2}(s)+O\left(s^{-1 / 2}\right) \text { as } s \rightarrow+\infty,
\end{aligned}
$$

$$
\text { for fixed } \sin ((|k|-k \hat{l}) \tau) \neq 0, \quad E, \tau \text { and } \lambda \text {, }
$$


Multipoint formulas for phase recovering

where $f_{1}=f_{1}(k, l), f_{2}=f_{2}(k, l), f_{1,3 / 2}(s)=f_{1,3 / 2}(k, l, \tau, \lambda, s)$, $f_{2,1 / 2}(s)=f_{2,1 / 2}(k, l, \tau, \lambda, s)$,

$$
\begin{aligned}
& f_{1,3 / 2}(s)=-\frac{z_{2,1}(s)}{\lambda-1}+\frac{\lambda z_{2,1}(\lambda s)}{\lambda-1}, \\
& f_{2,1 / 2}(s)=\frac{s \lambda}{\lambda-1}\left(z_{2,1}(s)-z_{2,1}(\lambda s)\right),
\end{aligned}
$$

where $z_{2,1}(s)=z_{2,1}(k, l, \tau, s)$ is found via $(9.2),(9.4),(10.2)$ from $\left|\psi^{+}(x, k)\right|^{2}$ at $x=x_{1}, x_{2}$ of $(6.1), z_{2,1}(\lambda s)=z_{2,1}(k, l, \tau, \lambda s)$ is found via (9.2), (9.4), (10.2) (where $s$ and $x_{1}, x_{2}$ are replaced by $\lambda s$ and $x_{3}, x_{4}$ of (7.1) for $\left.n=2\right),(k, l) \in \Omega_{E}, d=2, E>0, \hat{l}=l /|l|$, $\tau>0, \lambda>1$.

Let $f_{1,1 / 2}(\kappa s)=f_{1,1 / 2}(k, l, \tau, \kappa s)$ be defined by

$$
\begin{aligned}
& \left(\begin{array}{c}
f_{1,1 / 2}(\kappa s) \\
\bar{f}_{1,1 / 2}(\kappa s)
\end{array}\right)=M\left(\begin{array}{c}
a\left(y_{1}, k\right) \\
a\left(y_{2}, k\right),
\end{array}\right) \\
& y_{1}=\kappa s \hat{l}, \quad y_{2}=(\kappa s+\tau) \hat{l},
\end{aligned}
$$

where $M=M_{k, l, \tau, \kappa s}$ is defined according to (5.3), (5.4), (5.7), $a$ is defined by (2.1), $(k, l) \in \Omega_{E}, d=2, E>0, \hat{l}=l /|l|, s>0, \kappa \geq 1$.

Proposition 10.3 Under assumptions (1.2), (1.7) for $d=2$, the following formulas hold:

$$
\begin{aligned}
& z_{2}(\kappa s)=\zeta_{2,3 / 2}(\kappa, s)+O\left(s^{-2}\right) \text { as } s \rightarrow+\infty \\
& \text { for fixed } \sin ((|k|-k \hat{l}) \tau) \neq 0, E, \tau, \lambda \text { and } \kappa,
\end{aligned}
$$

where $z_{2}(s)=z_{2}(k, l, s)$ is defined by (5.1) for $n=2, \zeta_{2,3 / 2}(\kappa, s)=\zeta_{2,3 / 2}(k, l, \tau, \lambda, \kappa, s)$ is defined as follows:

$$
\begin{aligned}
& \left(\begin{array}{c}
\zeta_{2,3 / 2}(\kappa, s) \\
\bar{\zeta}_{2,3 / 2}(\kappa, s)
\end{array}\right)=\left(\begin{array}{c}
f_{1,1 / 2}(\kappa s) \\
\bar{f}_{1,1 / 2}(\kappa s)
\end{array}\right)-\left|f_{1,3 / 2}(s)\right|^{2} M\left(\begin{array}{c}
(\kappa s)^{-1 / 2} \\
(\kappa s+\tau)^{-1 / 2}
\end{array}\right)- \\
& \frac{\left(f_{1,3 / 2}(s) \bar{f}_{2,1 / 2}(s)+\bar{f}_{1,3 / 2}(s) f_{2,1 / 2}(s)\right)}{(\kappa s)^{3 / 2}} M\left(\begin{array}{l}
1 \\
1
\end{array}\right),
\end{aligned}
$$

$f_{1,1 / 2}(\kappa s)$ is defined by (10.10), $M=M_{k, l, \tau, \kappa s}$ is defined according to (5.3), (5.4), $(k, l) \in \Omega_{E}, d=2, E>0, \hat{l}=l /|l|, \tau>0, \kappa \geq 1$.

Proof of Proposition 10.3. Proposition 10.3 follows from Proposition 5.1 for $n=3$, $d=2$ and formulas (10.6), (10.7). In more detail, the proof consists of the following.

Formulas (8.1), (4.11), (4.13), (8.3) for $n=3$ imply that

$$
\begin{aligned}
& a_{3}^{2}(s \hat{l}, k)=\frac{\left|f_{1}\right|^{2}}{s^{1 / 2}}+\frac{f_{1} \bar{f}_{2}+\bar{f}_{1} f_{2}}{s^{3 / 2}}, \\
& \Delta_{3}^{1, \tau}(s \hat{l}, k)=\frac{e^{i \varphi_{\tau}} f_{2}}{s} w_{1}(s, \tau)=O\left(\frac{1}{s^{2}}\right), \quad s \rightarrow+\infty, \\
& a_{3}^{2}((s+\tau) \hat{l}, k)=\frac{\left|f_{1}\right|^{2}}{(s+\tau)^{1 / 2}}+\frac{f_{1} \bar{f}_{2}+\bar{f}_{1} f_{2}}{(s+\tau)^{3 / 2}},
\end{aligned}
$$


where $f_{1}=f_{1}(k, l), f_{2}=f_{2}(k, l), s>0, \tau>0$.

Formulas (5.2)-(5.4), (8.5), (5.6), (5.7) for $n=3$ and formulas (10.14)-(10.16) imply that

$$
\begin{aligned}
& \left(\begin{array}{c}
z_{3}(\kappa s) \\
\bar{z}_{3}(\kappa s)
\end{array}\right)=M\left(\begin{array}{c}
a(\kappa s \hat{l}, k) \\
a((\kappa s+\tau) \hat{l}, k)
\end{array}\right)-\left|f_{1}\right|^{2} M\left(\begin{array}{c}
(\kappa s)^{-1 / 2} \\
(\kappa s+\tau)^{-1 / 2}
\end{array}\right)- \\
& \left(f_{1} \bar{f}_{2}+\bar{f}_{1} f_{2}\right) M\left(\begin{array}{c}
(\kappa s)^{-3 / 2} \\
(\kappa s+\tau)^{-3 / 2}
\end{array}\right)+O\left(\frac{1}{(\kappa s)^{5 / 2}}\right) \text { as } s \rightarrow+\infty
\end{aligned}
$$

for fixed $\sin ((|k|-k \hat{l}) \tau) \neq 0, E$ and $\tau$,

where $M=M_{k, l, \tau, \kappa s}$.

Formula (5.1), for $n=2$ and for $n=3$, and formulas (10.10), (10.11), (10.6), (10.7), (10.17) imply (10.12), (10.13).

This completes the proof of Proposition 10.3.

Next, due to definition (5.1), for $n=2$, and formula (10.12), for $\kappa=1$ and for $\kappa=\lambda$, we have that

$$
\begin{aligned}
& f_{1}+s^{-1} f_{2}=\zeta_{2,3 / 2}(1, s)+O\left(s^{-2}\right) \text { as } s \rightarrow+\infty \\
& f_{1}+(\lambda s)^{-1} f_{2}=\zeta_{2,3 / 2}(\lambda, s)+O\left(s^{-2}\right) \text { as } s \rightarrow+\infty \\
& \text { for fixed } \sin ((|k|-k \hat{l}) \tau) \neq 0, E, \tau, \text { and } \lambda,
\end{aligned}
$$

where $f_{1}=f_{1}(k, l), f_{2}=f_{2}(k, l), \zeta_{2,3 / 2}(\kappa, s)=\zeta_{2,3 / 2}(k, l, \tau, \lambda, \kappa, s),(k, l) \in \Omega_{E}, d=2$, $E>0, \hat{l}=l /|l|, \tau>0$.

Proceeding from (10.18), (10.19), we obtain the following result.

Proposition 10.4. Under assumptions (1.2), (1.7) for $d=2$, the following formulas hold:

$$
\begin{aligned}
& f_{1}=f_{1,2}(s)+O\left(s^{-2}\right) \text { as } s \rightarrow+\infty \\
& f_{2}=f_{2,1}(s)+O\left(s^{-1}\right) \text { as } s \rightarrow+\infty \\
& \text { for fixed } \sin ((|k|-k \hat{l}) \tau) \neq 0, \quad E, \tau \text { and } \lambda,
\end{aligned}
$$

where $f_{1}=f_{1}(k, l), f_{2}=f_{2}(k, l), f_{1,2}(s)=f_{1,2}(k, l, \tau, \lambda, s)$, $f_{2,1}(s)=f_{2,1}(k, l, \tau, \lambda, s)$,

$$
\begin{aligned}
& f_{1,2}(s)=-\frac{\zeta_{2,3 / 2}(1, s)}{\lambda-1}+\frac{\lambda \zeta_{2,3 / 2}(\lambda, s)}{\lambda-1}, \\
& f_{2,1}(s)=\frac{s \lambda}{\lambda-1}\left(\zeta_{2,3 / 2}(1, s)-\zeta_{2,3 / 2}(\lambda, s)\right),
\end{aligned}
$$

where $\zeta_{2,3 / 2}(1, s)=\zeta_{2,3 / 2}(k, l, \tau, \lambda, 1, s), \zeta_{2,3 / 2}(\lambda, s)=\zeta_{2,3 / 2}(k, l, \tau, \lambda, \lambda, s)$ are found via (10.13), (10.10), (10.11), (10.8), (10.9) from $\left|\psi^{+}(x, k)\right|^{2}$ at $x=x_{1}, x_{2}, x_{3}, x_{4}$ of (6.1) for $n=2,(k, l) \in \Omega_{E}, d=2, E>0, \hat{l}=l /|l|, \tau>0, \lambda>1$.

11. Inductive finding $f_{1}, \ldots, f_{n}$ for the 2n-point case for $d=2$ 
Multipoint formulas for phase recovering

In this section we realize the scheme of Section 3 for the case when $d=2$ and $x_{1}, \ldots, x_{2 n}$ are defined as in (1.15).

11.1 Step of induction. Formulas (7.1)-(7.4) of Subsection 7.1 with $d=2$ in place of $d=3$ can be considered as the step of induction for $d=2$. However, for $d=2$ this step of induction consists of the following two substeps.

The first substep consists in finding $f_{1, n-1 / 2}, f_{2, n-1-1 / 2}, \ldots, f_{n, 1 / 2}$ proceeding from $f_{1, n-1}, f_{2, n-2}, \ldots, f_{n-1,1}$ of $(7.1),(7.2)$ for $d=2$, where

$$
\begin{aligned}
& f_{1, n-1 / 2}(s), f_{2, n-1-1 / 2}(s), \ldots, f_{n, 1 / 2}(s) \text { are functions found from } \\
& \left|\psi^{+}(x, k)\right|^{2} \text { given at } x=x_{1}(s), \ldots, x_{2 n}(s) \text { defined by }(1.15)
\end{aligned}
$$

and such that

$$
\begin{aligned}
& f_{j}=f_{j, n-j+1 / 2}(s)+O\left(s^{-(n-j+1 / 2)}\right), \quad s \rightarrow+\infty, \quad j=1, \ldots, n, \\
& \text { for fixed } \sin ((|k|-k \hat{l}) \tau) \neq 0, \quad E, \quad \tau \text { and } \lambda_{1}, \ldots, \lambda_{n},
\end{aligned}
$$

where $f_{j}=f_{j}(k, l), f_{j, n-j+1 / 2}(s)=f_{j, n-j+1 / 2}\left(k, l, \tau, \lambda_{1}, \ldots, \lambda_{n}, s\right),(k, l) \in \Omega_{E}, d=$ $2, E>0, \hat{l}=l /|l|, \tau>0$. For $n=2$ and $n=3$ the aforementioned functions $f_{1, n-1}, f_{2, n-2}, \ldots, f_{n-1,1}$ of $(7.1),(7.2)$ for $d=2$ are found in Sections 9 and 10.

This substep is realized via Theorems 11.1 and 11.2 formulated in the next subsection.

The second substep consists in finding $f_{1, n}, f_{2, n-1}, \ldots, f_{n, 1}$ proceeding from

$f_{1, n-1 / 2}, f_{2, n-1-1 / 2}, \ldots, f_{n, 1 / 2}$ of $(11.1)$, (11.2), where $f_{1, n}, f_{2, n-1}, \ldots, f_{n, 1}$ are the functions of $(7.3),(7.4)$ for $d=2$.

This substep is realized via Theorems 11.3 and 11.4 formulated in the next subsection.

11.2 Realization of the inductive step. Let $f_{1,1 / 2}(\kappa s)=f_{1,1 / 2}(k, l, \tau, \kappa s)$ be defined by (10.10), (10.11).

Let

$$
\begin{aligned}
& a_{n, n-1}^{2}(\kappa, s)=\sum_{\substack{j \in \mathbb{N} \\
2 j \leq n}} \frac{\left|f_{j, n-j}(s)\right|^{2}}{(\kappa s)^{2 j-1-1 / 2}}+\sum_{\substack{j_{1}, j_{2} \in \mathbb{N} \\
j_{1} \neq j_{2}, j_{1}+j_{2} \leq n}} \frac{f_{j_{1}, n-j_{1}}(s) \bar{f}_{j_{2}, n-j_{2}}(s)}{(\kappa s)^{j_{1}+j_{2}-1-1 / 2}}, \\
& a_{n, n-1}^{2, \tau}(\kappa, s)=\sum_{\substack{j \in \mathbb{N} \\
2 j \leq n}} \frac{\left|f_{j, n-j}(s)\right|^{2}}{(\kappa s+\tau)^{2 j-1-1 / 2}}+\sum_{\substack{j_{1}, j_{2} \in \mathbb{N} \\
j_{1} \neq j_{2}, j_{1}+j_{2} \leq n}} \frac{f_{j_{1}, n-j_{1}}(s) \bar{f}_{j_{2}, n-j_{2}}(s)}{(\kappa s+\tau)^{j_{1}+j_{2}-1-1 / 2}},
\end{aligned}
$$

where $f_{j, n-j}(s), j=1, \ldots, n-1$, are the functions of $(7.1),(7.2)$ for $d=2$.

Let $\Delta_{n, n-1}^{1, \tau}(\kappa, s)$ be defined by $(7.9)$ for $d=2$.

Theorem 11.1. Under assumptions (1.2), (1.7) for $d=2$, the following formulas hold:

$$
\begin{aligned}
& z_{n}(\kappa s)=\zeta_{n, n-1}(\kappa, s)+O\left(s^{-(n-1 / 2)}\right) \text { as } s \rightarrow+\infty \\
& \text { for fixed } \sin ((|k|-k \hat{l}) \tau) \neq 0, E, \tau \text { and } \lambda_{1}, \ldots, \lambda_{n-1}, \kappa,
\end{aligned}
$$

where $z_{n}(s)=z_{n}(k, l, s)$ is defined by (5.1), 
R.G. Novikov

$\zeta_{n, n-1}(\kappa, s)=\zeta_{n, n-1}\left(k, l, \tau, \lambda_{1}, \ldots, \lambda_{n-1}, \kappa, s\right)$ is defined as follows:

$$
\left(\begin{array}{c}
\zeta_{n, n-1}(\kappa, s) \\
\bar{\zeta}_{n, n-1}(\kappa, s)
\end{array}\right)=\left(\begin{array}{c}
f_{1,1 / 2}(\kappa s) \\
\bar{f}_{1,1 / 2}(\kappa s)
\end{array}\right)-M\left(\begin{array}{c}
a_{n, n-1}^{2}(\kappa, s) \\
\Delta_{n, n-1}^{1, \tau}(\kappa, s)+a_{n, n-1}^{2, \tau}(\kappa, s)
\end{array}\right),
$$

$f_{1,1 / 2}(\kappa s)$ is defined by (10.10), $M=M_{k, l, \tau, \kappa s}$ is defined according to (5.3), (5.4), $a_{n, n-1}^{2}(\kappa, s), a_{n, n-1}^{2, \tau}(\kappa, s)$ are defined by (11.3), (11.4), $\Delta_{n, n-1}^{1, \tau}(\kappa, s)$ is defined according to (7.9), $(k, l) \in \Omega_{E}, d=2, E>0, \hat{l}=l /|l|, \tau>0, \lambda_{1}, \ldots, \lambda_{n-1}$ are the numbers of (1.15), $\kappa \geq 1, n \geq 2$.

Theorem 11.2. Under assumptions (1.2), (1.7) for $d=2$, formulas (11.2) hold, where

$$
f_{i, n-i+1 / 2}(s)=s^{i-1} \sum_{j=1}^{n} \Lambda_{i, j}^{-1} \zeta_{n, n-1}\left(\lambda_{j}, s\right), \quad i=1, \ldots, n,
$$

where $\Lambda^{-1}=\left(\Lambda_{i, j}^{-1}\right)$ is the inverse of the matrix $\Lambda$ defined by $(7.12), \zeta_{n, n-1}(\kappa, s)$ is defined by (11.6), $n \geq 2$.

Note that $f_{1,3 / 2}, f_{2,1 / 2}$ of Theorem 11.2 for $n=2$ are different, in general, from $f_{1,3 / 2}$, $f_{2,1 / 2}$ of Proposition 10.2.

Theorems 11.1 and 11.2 realize the first substep of induction of Subsection 11.1.

Let

$$
\begin{aligned}
& a_{n+1, n-1 / 2}^{2}(\kappa, s)= \\
& \sum_{\substack{j \in \mathbb{N} \\
2 j \leq n+1}} \frac{\left|f_{j, n-j+1 / 2}(s)\right|^{2}}{(\kappa s)^{2 j-1-1 / 2}}+\sum_{\substack{j_{1}, j_{2} \in \mathbb{N} \\
j_{1} \neq j_{2}, j_{1}+j_{2} \leq n+1}} \frac{f_{j_{1}, n-j_{1}+1 / 2}(s) \bar{f}_{j_{2}, n-j_{2}+1 / 2}(s)}{(\kappa s)^{j_{1}+j_{2}-1-1 / 2}}, \\
& a_{n+1, n-1 / 2}^{2, \tau}(\kappa, s)= \\
& \sum_{\substack{j \in \mathbb{N} \\
2 j \leq n+1}} \frac{\left|f_{j, n-j+1 / 2}(s)\right|^{2}}{(\kappa s+\tau)^{2 j-1-1 / 2}}+\sum_{\substack{j_{1}, j_{2} \in \mathbb{N} \\
j_{1} \neq j_{2}, j_{1}+j_{2} \leq n+1}} \frac{f_{j_{1}, n-j_{1}+1 / 2}(s) \bar{f}_{j_{2}, n-j_{2}+1 / 2}(s)}{(\kappa s+\tau)^{j_{1}+j_{2}-1-1 / 2}},
\end{aligned}
$$

where $f_{j, n-j+1 / 2}(s), j=1, \ldots, n$, are the functions of (11.1), (11.2), (11.7). hold:

Theorem 11.3. Under assumptions (1.2), (1.7) for $d=2$, the following formulas

$$
\begin{aligned}
& z_{n}(\kappa s)=\zeta_{n, n-1 / 2}(\kappa, s)+O\left(s^{-n}\right) \text { as } s \rightarrow+\infty \\
& \text { for fixed } \sin ((|k|-k \hat{l}) \tau) \neq 0, E, \tau \text { and } \lambda_{1}, \ldots, \lambda_{n}, \kappa,
\end{aligned}
$$

where $z_{n}(s)=z_{n}(k, l, s)$ is defined by (5.1), $\zeta_{n, n-1 / 2}(\kappa, s)=\zeta_{n, n-1}\left(k, l, \tau, \lambda_{1}, \ldots, \lambda_{n}, \kappa, s\right)$ is defined as follows:

$$
\left(\begin{array}{c}
\zeta_{n, n-1 / 2}(\kappa, s) \\
\bar{\zeta}_{n, n-1 / 2}(\kappa, s)
\end{array}\right)=\left(\begin{array}{c}
f_{1,1 / 2}(\kappa s) \\
\bar{f}_{1,1 / 2}(\kappa s)
\end{array}\right)-M\left(\begin{array}{c}
a_{n+1, n-1 / 2}^{2}(\kappa, s) \\
\Delta_{n, n-1}^{1, \tau}(\kappa, s)+a_{n+1, n-1 / 2}^{2, \tau}(\kappa, s)
\end{array}\right)
$$


Multipoint formulas for phase recovering

$f_{1,1 / 2}(\kappa s)$ is defined by (10.10), $M=M_{k, l, \tau, \kappa s}$ is defined according to (5.3), (5.4), $a_{n+1, n-1 / 2}^{2}(\kappa, s), a_{n+1, n-1 / 2}^{2, \tau}(\kappa, s)$ are defined by (11.8), (11.9), $\Delta_{n, n-1}^{1, \tau}(\kappa, s)$ is defined according to (7.9), $(k, l) \in \Omega_{E}, d=2, E>0, \hat{l}=l /|l|, \tau>0, \lambda_{1}, \ldots, \lambda_{n}$ are the numbers of (1.15), $\kappa \geq 1, n \geq 2$.

Theorem 11.4. Under assumptions (1.2), (1.7) for $d=2$, formulas (7.4) for $d=2$ hold, where

$$
f_{i, n-i+1}(s)=s^{i-1} \sum_{j=1}^{n} \Lambda_{i, j}^{-1} \zeta_{n, n-1 / 2}\left(\lambda_{j}, s\right), \quad i=1, \ldots, n,
$$

where $\Lambda^{-1}=\left(\Lambda_{i, j}^{-1}\right)$ is the inverse of the matrix $\Lambda$ defined by $(7.12), \zeta_{n, n-1 / 2}(\kappa, s)$ is defined by (11.11), $n \geq 2$.

Note that $\zeta_{2,3 / 2}$ and $f_{1,2}, f_{2,1}$ of Theorems 11.3 and 11.4 for $n=2$ are different, in general, from $\zeta_{2,3 / 2}$ and $f_{1,2}, f_{2,1}$ of Propositions 10.3 and 10.4.

Theorems 11.3 and 11.4 realize the second substep of induction of Subsection 11.1.

11.3. Proofs of Theorems 11.1-11.4.

Proof of Theorem 11.1. Theorem 11.1 follows from Proposition 5.1 for $d=2$ with formulas (8.5) in place of (5.5), formula (10.10) and the formulas

$$
\begin{aligned}
& a_{n}^{2}(\kappa s)=a_{n, n-1}^{2}(\kappa, s)+O\left(s^{-(n-1 / 2)}\right), \quad s \rightarrow+\infty, \\
& a_{n}^{2}(\kappa s+\tau)=a_{n, n-1}^{2, \tau}(\kappa, s)+O\left(s^{-(n-1 / 2)}\right), \quad s \rightarrow+\infty, \\
& \Delta_{n}^{1, \tau}(\kappa s)=\Delta_{n, n-1}^{1, \tau}(\kappa, s)+O\left(s^{-n}\right), \quad s \rightarrow+\infty, \\
& \text { for fixed } \sin ((|k|-k \hat{l}) \tau) \neq 0, E, \tau \text { and } \lambda_{1}, \ldots, \lambda_{n-1}, \kappa,
\end{aligned}
$$

where $a_{n}^{2}(\kappa s)=a_{n}^{2}(\kappa s \hat{l}, k), a_{n}^{2}(\kappa s+\tau)=a_{n}^{2}((\kappa s+\tau) \hat{l}, k)$ are the functions of (8.1), (8.3), $\Delta_{n}^{1, \tau}(\kappa s)=\Delta_{n}^{1, \tau}(\kappa s \hat{l}, k)$ is the function of (4.11) for $d=2, a_{n, n-1}^{2}(\kappa, s), a_{n, n-1}^{2, \tau}(\kappa, s)$ are the functions of (11.3), (11.4), $\Delta_{n, n-1}^{1, \tau}(\kappa, s)$ is the function of $(7.9)$ for $d=2$.

Formula (11.13) follows from $(8.1),(11.3),(7.2)$ for $d=2$, and the observation that the terms

$$
\begin{aligned}
& O\left(s^{-(n+j-1-1 / 2)}\right), O\left(s^{-\left(n+j_{1}-1-1 / 2\right)}\right), O\left(s^{-\left(n+j_{2}-1-1 / 2\right)}\right), \\
& j \in \mathbb{N}, 2 j \leq n, j_{1}, j_{2} \in \mathbb{N}, j_{1} \neq j_{2}, j_{1}+j_{2} \leq n,
\end{aligned}
$$

arising at comparison of the summands of $a_{n}^{2}(\kappa s)$ and $a_{n, n-1}^{2}(\kappa, s)$ are majorized by $O\left(s^{-(n-1 / 2)}\right)$ as $s \rightarrow+\infty$.

Formula (11.14) is proved in a similar way with formula (11.13).

Formula (11.15) for $d=2$ is completely similar to formula (7.17) for $d=3$.

This completes the proof of Theorem 11.1.

Proof of Theorem 11.2. Using formula (5.1) for $d=2$ and formula (11.5) we obtain (7.18)-(7.22) for $d=2$, where $O\left(s^{-n}\right)$ is replaced by $O\left(s^{-(n-1 / 2)}\right)$ in $(7.18),(7.21),(7.22)$.

Formulas (11.2), (11.7) follow from (7.19), (7.20), (7.22) for $d=2$, where $O\left(s^{-n}\right)$ is replaced by $O\left(s^{-(n-1 / 2)}\right)$ in $(7.22)$.

Theorem 11.2 is proved. 
Proof of Theorem 11.3. Due to formulas (5.1)-(5.4), (5.6), (5.7) for $d=2$ and formulas (8.5), (10.10), (10.11) we have that

$$
\begin{aligned}
& \left(\begin{array}{l}
z_{n+1}(\kappa s) \\
\bar{z}_{n+1}(\kappa s)
\end{array}\right)=\left(\begin{array}{c}
f_{1,1 / 2}(\kappa s) \\
\bar{f}_{1,1 / 2}(\kappa s)
\end{array}\right)- \\
& M\left(\left(\begin{array}{c}
a_{n+1}^{2}(\kappa s) \\
\Delta_{n+1}^{1, \tau}(\kappa s)+a_{n+1}^{2}(\kappa s+\tau)
\end{array}\right)+\left(\begin{array}{c}
\delta_{n+1} a(\kappa s) \\
\rho_{n+1}^{1, \tau}(\kappa s)+\delta_{n+1} a(\kappa s+\tau)
\end{array}\right)\right) \\
& \delta_{n+1} a(\kappa s)=O\left(s^{-n-1 / 2}\right), \quad \delta_{n+1} a(\kappa s+\tau)=O\left(s^{-n-1 / 2}\right) \text { as } s \rightarrow+\infty \\
& \rho_{n+1}^{1, \tau}(\kappa s)=O\left(s^{-n-1}\right) \text { as } s \rightarrow+\infty
\end{aligned}
$$

uniformly in $\hat{k}, \hat{l}$ at fixed $\tau>0$ and $E>0$, where $z_{n+1}(\kappa s)=z_{n+1}(k, l, \kappa s)$, $f_{1,1 / 2}(\kappa s)=f_{1,1 / 2}(k, l, \tau, \kappa s), M=M_{k, l, \tau, \kappa s}, a_{n+1}^{2}(\kappa s)=a_{n+1}^{2}(\kappa s \hat{l}, k)$, $a_{n+1}^{2}(\kappa s+\tau)=a_{n+1}^{2}((\kappa s+\tau) \hat{l}, k), \Delta_{n+1}^{1, \tau}(\kappa s)=\Delta_{n+1}^{1, \tau}(\kappa s \hat{l}, k), \delta_{n+1} a(\kappa s)=\delta_{n+1} a(\kappa s \hat{l}, k)$, $\rho_{n+1}^{1, \tau}(\kappa s)=\rho_{n+1}^{1, \tau}(\kappa s \hat{l}, k), \delta_{n+1} a(\kappa s+\tau)=\delta_{n+1} a((\kappa s+\tau) \hat{l}, k),(k, l) \in \Omega_{E}, d=2, E>0$, $\hat{k}=k /|k|, \hat{l}=l /|l|, \tau>0, n \in \mathbb{N}, \kappa \geq 1, \sin ((|k|-k \hat{l}) \tau) \neq 0$.

In addition, using $(4.11),(4.13),(5.1)$ for $d=2$, one can see that

$$
\begin{aligned}
& z_{n+1}(\kappa s)=z_{n}(\kappa s)+O\left(s^{-n}\right), \quad s \rightarrow+\infty, \\
& \Delta_{n+1}^{1, \tau}(\kappa s)=\Delta_{n}^{1, \tau}(\kappa s)+O\left(s^{-n}\right), \quad s \rightarrow+\infty,
\end{aligned}
$$

uniformly in $\hat{k}, \hat{l}$ and $\kappa \geq 1$ at fixed $\tau>0$ and $E>0$.

Besides, the following formulas hold:

$$
\begin{aligned}
& a_{n+1}^{2}(\kappa s)=a_{n+1, n-1 / 2}^{2}(\kappa, s)+O\left(s^{-n}\right), \quad s \rightarrow+\infty, \\
& a_{n+1}^{2}(\kappa s+\tau)=a_{n+1, n-1 / 2}^{2, \tau}(\kappa, s)+O\left(s^{-n}\right), \quad s \rightarrow+\infty,
\end{aligned}
$$

for fixed $\sin ((|k|-k \hat{l}) \tau) \neq 0, E, \tau$ and $\lambda_{1}, \ldots, \lambda_{n}, \kappa$, where $a_{n+1}^{2}(\kappa s), a_{n+1}^{2}(\kappa s+\tau)$ are the functions arising in (11.16), $a_{n+1, n-1 / 2}^{2}(\kappa, s)$, $a_{n+1, n-1 / 2}^{2, \tau}(\kappa, s)$ are defined by (11.8), (11.9).

Formula (11.21) follows from (8.1) with $n+1$ in place of $n,(11.8),(11.2)$ and the observation that the terms

$$
\begin{aligned}
& O\left(s^{-(n+j-1)}\right), \quad O\left(s^{-\left(n+j_{1}-1\right)}\right), \quad O\left(s^{-\left(n+j_{2}-1\right)}\right), \\
& j \in \mathbb{N}, \quad 2 j \leq n+1, \quad j_{1}, j_{2} \in \mathbb{N}, \quad j_{1} \neq j_{2}, \quad j_{1}+j_{2} \leq n+1,
\end{aligned}
$$

arising at comparison of the summands of $a_{n+1}^{2}(\kappa s)$ and $a_{n+1, n-1 / 2}^{2}(\kappa, s)$ are majorized by $O\left(s^{-n}\right)$ as $s \rightarrow+\infty$.

Formulas (11.10), (11.11) follow from (11.16), (11.19), (11.17), (11.18), (11.21), (11.22) and (11.20), (11.15).

This completes the proof of Theorem 11.3.

Proof of Theorem 11.4. Using formula (5.1) for $d=2$ and formula (11.10) we obtain (7.18)-(7.22) for $d=2$, where $\zeta_{n, n-1}$ is replaced by $\zeta_{n, n-1 / 2}$. 
Multipoint formulas for phase recovering

Formulas (7.4) for $d=2$, (11.12) follow from (7.19), (7.20), (7.22), where $\zeta_{n, n-1}$ is replaced by $\zeta_{n, n-1 / 2}$ in $(7.20)$.

Theorem 11.4 is proved.

\section{Formulas for $\psi^{+}$}

Theorem 12.1. Under assumptions (1.2), (1.7) for $d=3$ and for $d=2$, the following formulas hold:

$$
\begin{aligned}
& \psi^{+}(s \hat{l}, k)=\tilde{\psi}_{n}^{+}(s)+O\left(s^{-n-(d-1) / 2}\right) \text { as } s \rightarrow+\infty \\
& \text { for fixed } \sin ((|k|-k \hat{l}) \tau) \neq 0, E, \tau \text { and } \lambda_{1}, \ldots, \lambda_{n}
\end{aligned}
$$

where $\psi^{+}$is the function of (1.4), (3.3), $\tilde{\psi}_{n}^{+}(s)=\tilde{\psi}_{n}^{+}\left(k, l, \tau, \lambda_{1}, \ldots, \lambda_{n}, s\right)$ is found from $\left|\psi^{+}(x, k)\right|^{2}$ given at $x=x_{1}(s), \ldots, x_{2 n}(s)$ defined by (1.15),

$$
\tilde{\psi}_{n}^{+}(s)=e^{i s k \hat{l}}+\frac{e^{i|k| s}}{s^{(d-1) / 2}}\left(\sum_{j=1}^{n} \frac{f_{j, n-j+1}(s)}{s^{j-1}}\right),
$$

where $f_{j, n-j+1}, j=1, \ldots, n$, are the functions of (7.3), (7.4) (see Section 7 for $d=3$ and Section 11 for $d=2),(k, l) \in \Omega_{E}, E>0, \hat{l}=l /|l|, n \in \mathbb{N}$.

Formulas (12.1) follow from expansion (3.3), definition (12.2) and the asymptotic formulas (7.4).

Theorem 12.1 realizes formulas (1.16), (3.5).

Acknowledgements The author thanks B. Berndtsson and M.V. Klibanov for remarks on results of [N2], [N4], which stimulated studies of the present work.

\section{References}

[AHN] A.D. Agaltsov, T. Hohage, R.G. Novikov, An iterative approach to monochromatic phaseless inverse scattering, Inverse Problems 35(2), 24001 ( 24 pp.) (2019)

[ B] M. Born, Quantenmechanik der Stossvorgange, Zeitschrift fur Physik 38 (11-12), 803$827(1926)$

[ChS] K. Chadan, P.C. Sabatier, Inverse Problems in Quantum Scattering Theory, 2nd edn. Springer, Berlin, 1989.

[ FM] L.D. Faddeev, S.P. Merkuriev, Quantum Scattering Theory for Multi-particle Systems, Mathematical Physics and Applied Mathematics, 11. Kluwer Academic Publishers Group, Dordrecht, 1993

[ IK] O. Ivanyshyn, R. Kress, Identification of sound-soft 3D obstacles from phaseless data, Inverse Probl. Imaging 4, 131-149 (2010)

[ JL] P. Jonas, A.K. Louis, Phase contrast tomography using holographic measurements, Inverse Problems 20(1), 75-102 (2004)

[ HN] T. Hohage, R.G. Novikov, Preface - Inverse wave propagation problems without phase information, Inverse Problems (to appear)

[K1] M.V. Klibanov, Phaseless inverse scattering problems in three dimensions, SIAM J.Appl. Math. 74(2), 392-410 ( 2014) 
[ K2] M.V. Klibanov, N.A. Koshev, D.-L. Nguyen, L.H. Nguyen, A. Brettin, V.N. Astratov, A numerical method to solve a phaseless coefficient inverse problem from a single measurement of experimental data. SIAM J. Imaging Sci. 11(4), 2339-2367 (2018)

[ KR] M.V. Klibanov, V.G. Romanov, Reconstruction procedures for two inverse scattering problems without the phase information, SIAM J. Appl. Math. 76(1), 178-196 (2016)

[ M] R.B. Melrose, Geometric scattering theory. Stanford Lectures. Cambridge University Press, 1995.

[ N1] R. G. Novikov, An iterative approach to non-overdetermined inverse scattering at fixed energy, Sbornik: Mathematics 206(1), 120-134 (2015)

[ N2] R. G. Novikov, Formulas for phase recovering from phaseless scattering data at fixed frequency, Bulletin des Sciences Mathématiques 139(8), 923-936 (2015)

[ N3] R. G. Novikov, Phaseless inverse scattering in the one-dimensional case, Eurasian Journal of Mathematical and Computer Applications 3(1), 63-69 (2015)

[ N4] R. G. Novikov, Inverse scattering without phase information, Séminaire Laurent Schwartz - EDP et applications (2014-2015), Exp. No16, 13p.

[ NG] R. G. Novikov, M.A. Galchenkova, Phase recovering from phaseless scattering data at a few points, Report of stage M2, June 2018 (unpublished)

[ P] V. Palamodov, A fast method of reconstruction for X-ray phase contrast imaging with arbitrary Fresnel number, arXiv:1803.08938v1 (2018)

[ R] V. G. Romanov, Inverse problems without phase information that use wave interference, Sib. Math. J. 59(3), 494-504 (2018) 